\title{
Mixed normal-superconducting states in the presence of strong electric currents
}

\author{
Yaniv Almog, Bernard Helffer $\stackrel{\dagger}{\dagger}$ and Xing-Bin Pan $\ddagger$
}

\begin{abstract}
We study the Ginzburg-Landau equations in the presence of large electric currents, that are smaller than the critical current where the normal state losses its stability. For steady-state solutions in the large $\kappa$ limit, we prove that the superconductivity order parameter is exponentially small in a significant part of the domain, and small in the rest of it. Similar results are obtained for the timedependent problem, in continuation of the paper by the two first authors [3]. We conclude by obtaining some weaker results, albeit similar, for steady-state solutions in the large domain limit.
\end{abstract}

\section{Contents}

1 Introduction $\quad 2$

2 Magnetic Laplacian Ground States 9

3 Steady-State Solutions 12

4 Time-Dependent Analysis $\quad 25$

$5 \quad$ Large domains 42

${ }^{*}$ Department of Mathematics, Louisiana State University, Baton Rouge, LA 70803, USA. almog@math.lsu.edu

${ }^{\dagger}$ Laboratoire de Mathématiques, Université Paris-Sud 11 and CNRS, Bât 425, 91405 Orsay Cedex, France and Laboratoire de Mathématiques Jean Leray, Université de Nantes.

Bernard.Helffer@math.u-psud.fr

${ }^{\ddagger}$ Department of Mathematics, East China Normal University, and NYU-ECNU Institute of Mathematical Sciences at NYU Shanghai, Shanghai 200062, P.R. China. xbpan@math.ecnu.edu.cn 


\section{Introduction}

Consider the time-dependent Ginzburg-Landau system of equations

$$
\begin{aligned}
\frac{\partial \psi}{\partial t}-\nabla_{\kappa A}^{2} \psi+i \kappa \phi \psi=\kappa^{2}\left(1-|\psi|^{2}\right) \psi & \text { in }(0,+\infty) \times \Omega, \\
\frac{1}{c}\left(\frac{\partial A}{\partial t}+\nabla \phi\right)+\operatorname{curl}^{2} A=\frac{1}{\kappa} \operatorname{Im}\left(\bar{\psi} \nabla_{\kappa A} \psi\right) & \text { in }(0,+\infty) \times \Omega, \\
\psi=0 & \text { on }(0,+\infty) \times \partial \Omega_{c}, \\
\nabla_{\kappa A} \psi \cdot \nu=0 & \text { on }(0,+\infty) \times \partial \Omega_{i}, \\
\frac{\partial \phi}{\partial \nu}=-c \kappa J(x) & \text { on }(0,+\infty) \times \partial \Omega_{c}, \\
\frac{\partial \phi}{\partial \nu}=0 & \text { on }(0,+\infty) \times \partial \Omega_{i}, \\
f_{\partial \Omega} \operatorname{curl} A(t, x) d s=\kappa h_{e x}, & \text { on }(0,+\infty) \\
\psi(0, x)=\psi_{0}(x) & \text { in } \Omega, \\
A(0, x)=A_{0}(x) & \text { in } \Omega .
\end{aligned}
$$

In the above $\psi$ denotes the order parameter, $A$ the magnetic potential, $\phi$ the electric potential, $\kappa$ the Ginzburg-Landau parameter, which is a material property, and

$$
c=\kappa^{2} / \sigma
$$

where $\sigma$ is the normal conductivity of the sample. Finally, $h_{e x}$, or the average magnetic field on $\partial \Omega$ divided by $\kappa$, is constant in time. Length has been scaled with respect to the penetration depth (see [3]). Unless otherwise stated we shall assume in the sequel that

$$
\kappa \geq 1 \text {. }
$$

We further assume that $\left(\psi_{0}, A_{0}\right) \in H^{1}(\Omega, \mathbb{C}) \times H^{1}\left(\Omega, \mathbb{R}^{2}\right)$ and that

$$
\left\|\psi_{0}\right\|_{\infty} \leq 1 \text {. }
$$

We use the notation $\nabla_{A}=\nabla-i A, \Delta_{A}=\nabla_{A}^{2}$ and $d s$ for the induced measure on $\partial \Omega$. We have also used above the standard notation

$$
f_{\partial \Omega}=\frac{1}{\ell(\partial \Omega)} \int
$$


The domain $\Omega \subset \subset \mathbb{R}^{2}$ has the same characteristics as in [3], in particular its boundary $\partial \Omega$ contains a smooth interface, denoted by $\partial \Omega_{c}$, with a conducting metal which is at the normal state. Thus, we require that $\psi$ vanishes on $\partial \Omega_{c}$ in $(1.1 \mathrm{c})$.

We make the following assumptions on the current $J$

$$
\begin{gathered}
(J 1) \quad J \in C^{2}\left(\overline{\partial \Omega_{c}}, \mathbb{R}\right), \\
(J 2) \quad \int_{\partial \Omega_{c}} J d s=0,
\end{gathered}
$$

and

(J3) the sign of $J$ is constant on each connected component of $\partial \Omega_{c}$.

We allow for $J \neq 0$ at the corners despite the fact that no current is allowed to enter the sample through the insulator. The rest of $\partial \Omega$, denoted by $\partial \Omega_{i}$ is adjacent to an insulator. By convention, we extend $J$ as equal to 0 on $\partial \Omega_{i}$.

To simplify some of our regularity arguments we introduce the following geometrical assumption (for further discussion we refer the reader to Appendix $\mathrm{A}$ in [3]) on $\partial \Omega$ :

$$
(R 1)\left\{\begin{array}{l}
(a) \partial \Omega_{i} \text { and } \partial \Omega_{c} \text { are of class } C^{3} ; \\
(b) \text { Near each edge, } \partial \Omega_{i} \text { and } \partial \Omega_{c} \text { are } \\
\text { flat and meet with an angle of } \frac{\pi}{2}
\end{array}\right.
$$

We also require that

(R2) Both $\partial \Omega_{c}$ and $\partial \Omega_{i}$ have two components.

Figure 1 presents a typical sample with properties (R1) and (R2), where the current flows into the sample from one connected component of $\partial \Omega_{c}$, and exits from another part, disconnected from the first one. Most wires would fall into the above class of domains.

The system (1.1) is invariant to the gauge transformation

$$
A^{\prime}=A+\nabla \omega, \phi^{\prime}=\phi-\frac{\partial \omega}{\partial t}, \psi^{\prime}=\psi e^{i \omega} .
$$




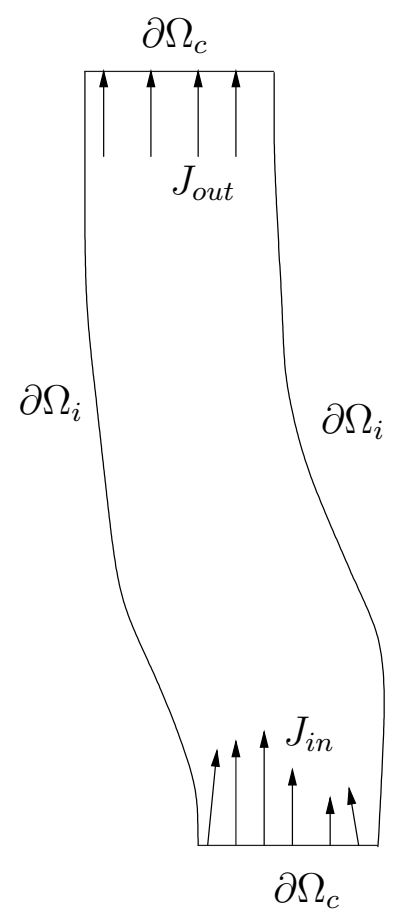

Figure 1: Typical superconducting sample. The arrows denote the direction of the current flow ( $J_{\text {in }}$ for the inlet, and $J_{\text {out }}$ for the outlet).

We thus choose, as in [3], the Coulomb gauge, i.e., we assume

$$
\begin{cases}\operatorname{div} A=0 & \text { in }(0,+\infty) \times \Omega, \\ A \cdot \nu=0 & \text { on }(0,+\infty) \times \partial \Omega,\end{cases}
$$

where the divergence is computed with respect to the spatial coordinates only.

From (3.1b), (3.1d), and (3.1f), we know that $\operatorname{curl} A$ is constant on each connected component of $\partial \Omega_{i}$. Let then $\left\{\partial \Omega_{i, j}\right\}_{j=1}^{2}$ denote the set of connected components of $\partial \Omega_{i}$. We can write, for $j=1,2$,

$$
\left.\operatorname{curl} A\right|_{\partial \Omega_{i, j}}=h_{j} \kappa,
$$

where $h_{1}$ and $h_{2}$ are constants.

Note that $h_{1}$ and $h_{2}$ can be determined from $J$ and $h_{e x}$ via the formula $[3]$

$$
h_{j}=h_{e x}-f_{\partial \Omega}\left|\Gamma\left(\tilde{x}, x_{j}\right)\right| J(\tilde{x}) d s(\tilde{x}) \text { for any } x_{j} \in \partial \Omega_{i, j}, \quad j=1,2,
$$


where $\Gamma\left(x, x_{0}\right)$ is the portion of $\partial \Omega$ connecting $x_{0}$ and $x$ in the positive trigonometric direction. We assume that $h_{e x}$ and $J$ are such that

$$
h_{1} h_{2}<0,
$$

and without any loss of generality we can assume $h_{2}>0$. Let

$$
h=\max \left(\left|h_{1}\right|,\left|h_{2}\right|\right) .
$$

We assume that

$$
h>1
$$

and distinguish below between two different cases

$$
1<h \leq \frac{1}{\Theta_{0}} \quad \text { or } \quad \frac{1}{\Theta_{0}}<h,
$$

where $\Theta_{0}$ is given by $(2.2)\left(\Theta_{0} \sim 0.59\right)$.

In [3] we have established the global existence of solutions for (1.1), such that

$$
\begin{aligned}
& \psi_{c} \in C\left([0,+\infty) ; W^{1+\alpha, 2}(\Omega, \mathbb{C})\right) \cap H_{\mathrm{loc}}^{1}\left([0,+\infty) ; L^{2}(\Omega, \mathbb{C})\right), \forall \alpha<1, \\
& A_{c} \in C\left([0,+\infty) ; W^{1, p}\left(\Omega, \mathbb{R}^{2}\right)\right) \cap H_{\mathrm{loc}}^{1}\left([0,+\infty) ; L^{2}\left(\Omega, \mathbb{R}^{2}\right)\right), \forall p \geq 1, \\
& \phi_{c} \in L_{\mathrm{loc}}^{2}\left([0,+\infty) ; H^{1}(\Omega)\right) .
\end{aligned}
$$

We next define, as in [3, Subsection 2.2], (in a slightly different manner as the definition here is $c$-independent) the normal fields. They are defined as the weak solution - $\left(\phi_{n}, A_{n}\right) \in H^{1}(\Omega) \times H^{1}\left(\Omega, \mathbb{R}^{2}\right)$ - of $(1.9)$ and

$$
\begin{cases}\operatorname{curl}^{2} A_{n}+\nabla \phi_{n}=0 & \text { in } \Omega \\ -\frac{\partial \phi_{n}}{\partial \nu}=J & \text { on } \partial \Omega \\ f_{\partial \Omega} \operatorname{curl} A_{n} d s=h_{e x}, & \\ \int_{\Omega} \phi_{n} d x=0 . & \end{cases}
$$


Note that $\left(0, \kappa A_{n}, c \kappa \phi_{n}\right)$ is a steady-state solution of (1.1). By (1.16) we have (cf. [3])

$$
\begin{cases}\Delta B_{n}=0 & \text { in } \Omega, \\ \frac{\partial B_{n}}{\partial \tau}=J & \text { on } \partial \Omega, \\ f_{\partial \Omega} B_{n}(x) d s=h_{e x} . & \end{cases}
$$

By taking the divergence of (1.16a) we also obtain

$$
\begin{cases}\Delta \phi_{n}=0 & \text { in } \Omega, \\ -\frac{\partial \phi_{n}}{\partial \nu}=J & \text { on } \partial \Omega, \\ \int_{\Omega} \phi_{n} d x=0 . & \end{cases}
$$

We recall from $[3,(2.16)$ and $(2.17)]$ that

$$
B_{n} \in W^{2, p}(\Omega), \phi_{n} \in W^{2, p}(\Omega), \forall p>1 .
$$

We focus attention in this work on the exponential decay of $\psi$ in regions where $\left|B_{n}\right|>1$. For steady-state solutions of (1.1) in the absence of electric current $(J=0)$ we may set $\phi \equiv 0$ and the magnetic field is then constant on the boundary. The exponential decay of $\psi$ away from the boundary has been termed "surface superconductivity" and has extensively been studied (cf. [11] and the references within). More recently, the case of a non-constant magnetic field has been studied as well $[4,14]$. In these works $\phi$ still identically vanishes but nevertheless $\nabla B_{n} \neq 0$ in view of the presence of a current source term curl $h_{e x}$ in (1.1b). In particular in [14] it has been established, in the large $\kappa$ limit for the case $1 \ll h \ll \kappa$ that $\psi$ is exponentially small away from $B_{n}^{-1}(0)$.

In the absence of electric current the time-dependent case is of lesser interest, since every solution of (1.1) converges to a steady-state solution $[17,10]$. This result has been obtained in [10] by using the fact that the Ginzburg-Landau energy functional is a Lyapunov function in this case. In contrast, when $J \neq 0$ this property of the energy functional is lost, and convergence to a steady-state is no-longer guaranteed. In [3] the global stability of the normal state has been established for $h=\mathcal{O}(\kappa)$ 
in the large $\kappa$ limit. In the present contribution, for the same limit, we explore the behavior of the solution for $1<h \ll \kappa$, and establish exponential decay of $\psi$ in every subdomain of $\Omega$ where $\left|B_{n}\right|>1$. We do that for both steady-state solutions (whose existence we need to assume) and time-dependent ones. We also study the large-domain limit, where we obtain weaker results for steady-state solutions only.

Let, for $j=1,2$,

$$
\omega_{j}=\left\{x \in \Omega:(-1)^{j} B_{n}(x)>1\right\} .
$$

Our first theorem concerns steady state solutions and their exponential decay, in certain subdomains of $\Omega$, in the large $\kappa$ limit.

Theorem 1.1. Let for $\kappa \geq 1,\left(\psi_{\kappa}, A_{\kappa}, \phi_{\kappa}\right)$ be a time-independent solution of (1.1). Suppose that for some $j \in\{1,2\}$ we have

$$
1<\left|h_{j}\right| \text {. }
$$

Then, for any compact set $K \subset \omega_{j} \cup \partial \Omega_{c}$, there exist $C>0, \alpha>0$, and $\kappa_{0} \geq 1$, such that for any $\kappa \geq \kappa_{0}$ we have

$$
\int_{K}\left|\psi_{\kappa}(x)\right|^{2} d x \leq C e^{-\alpha \kappa}
$$

If, in addition,

$$
\frac{1}{\Theta_{0}}<\left|h_{j}\right|
$$

then (1.22) is satisfied for any compact subset $K \subset \overline{\omega_{j}}$.

In addition to the above exponential decay, which is limited to the region where the normal magnetic field is large, we establish a weaker decay of $\psi_{\kappa}$ in the entire domain.

Proposition 1.2. Under the assumptions (1.3)-(1.15) there exists $C(J, \Omega)>$ 0 such that, for $\kappa \geq 1$,

$$
\left\|\psi_{\kappa}\right\|_{2} \leq C(J, \Omega)\left(1+c^{-1 / 2}\right)^{1 / 3} \kappa^{-1 / 6} .
$$

Theorem 1.1 is extended to the time dependent case in the following way. 
Theorem 1.3. Let $\left(\psi_{\kappa}, A_{\kappa}, \phi_{\kappa}\right)$ denote a time-dependent solution of (1.1). Assuming $c=1$, under the conditions of Theorem 1.1 and (1.2), for any compact set $K \subset \omega_{j} \cup \partial \Omega_{c}$ there exist $C>0, \alpha>0$, and $\kappa_{0} \geq 1$, such that for any $\kappa \geq \kappa_{0}$ we have

$$
\limsup _{t \rightarrow \infty} \int_{K}\left|\psi_{\kappa}(t, x)\right|^{2} d x \leq C e^{-\alpha \kappa} .
$$

Finally, we consider steady-state solutions of (1.1) in the large domain limit, i.e., we set $\kappa=c=1$ and stretch $\Omega$ by a factor of $R \gg 1$. Let $\Omega^{R}$ be the image of $\Omega$ under the map $x \rightarrow R x$. We consider again steady-state solutions of (1.1).

$$
\begin{array}{lr}
\Delta_{A} \psi+\psi\left(1-|\psi|^{2}\right)-i \phi \psi=0 & \text { in } \Omega^{R}, \\
\operatorname{curl}^{2} A+\nabla \phi=\operatorname{Im}\left(\bar{\psi} \nabla_{A} \psi\right) & \text { in } \Omega^{R}, \\
\psi=0 & \text { on } \partial \Omega_{c}^{R}, \\
\nabla_{A} \psi \cdot \nu=0 & \text { on } \partial \Omega_{i}^{R}, \\
\frac{\partial \phi}{\partial \nu}=\frac{F(R)}{R} J & \text { on } \partial \Omega_{c}^{R}, \\
\frac{\partial \phi}{\partial \nu}=0 & \text { on } \partial \Omega_{i}^{R}, \\
f_{\partial \Omega^{R}} \operatorname{curl} A(x) d s=F(R) h_{e x} . &
\end{array}
$$

In the above $F(R)=R^{\gamma}$ for some $0<\gamma<1$. We study the above problem in the limit $R \rightarrow \infty$. Assuming again (1.3)-(1.15) we establish the following result.

Proposition 1.4. Let $(\psi, A, \phi)$ denote a solution of (1.26). Then, there exists a compact set $K \subset \Omega, C>0, R_{0}>0$, and $\alpha>0$, such that for any $R>R_{0}$ we have

$$
\int_{K_{R}}|\psi(x)|^{2} d x \leq C e^{-\alpha R},
$$

where $K_{R}$ is the image of $K$ under the map $x \rightarrow R x$.

Note that $h_{e x}$ must be of $\mathcal{O}(J)$, otherwise $\left(0, A_{n}, \phi_{n}\right)$ would be the unique solution. 
Physically (1.27) demonstrates that there is a significant portion of the superconducting sample which remains, practically, at the normal state, for current densities which may be very small. This result stands in contrast with what one finds in standard physics handbooks [21] where the critical current density, for which the fully superconducting state looses its stability, is tabulated a material property. However, our results suggest that the critical current depends also on the geometry of the superconducting sample. In fact, according to Proposition 1.4, this current density must decay in the large domain limit. In two-dimensions, our result suggests that one should search for a critical current (and not current density), whereas in three-dimensions a density with respect to cross-section circumference (instead of area) should be obtained.

We note that Proposition 1.4 is certainly not optimal. In fact, we expect the following conjecture to be true.

Conjecture 1.5. Under the conditions of Proposition 1.4, for any compact set $K \subset \Omega \backslash B_{n}^{-1}(0)$, there exist $R_{0}>0, C>0$, and $\alpha>0$, such that for any $R>R_{0}(1.27)$ is satisfied.

The rest of this contribution is organized as follows. In the next section we establish some preliminary results related to the eigenvalues of the magnetic Laplacian in the presence of Dirichlet-Neumann corners. We use these results in Section 3 where we establish Theorem 1.1 and Proposition 1.2. In Section 4 we consider the time-dependent problem and establish, in particular, Theorem 1.3. Finally, in the last section, we obtain some weaker results for steady-state solutions of (1.1) in the large domain limit.

\section{Magnetic Laplacian Ground States}

In this section, we analyze the spectral properties of the Schrödinger operator with constant magnetic field in a sector. The Neumann problem has been addressed by V. Bonnaillie-Noël in [6]. In the sequel we shall need, however, a lower bound for the ground state energy of the above operator on a Dirichlet-Neumann sector, i.e., a Dirichlet condition is prescribed on one side of the sector and the magnetic Neumann condition 
on the other side. We begin by the following auxiliary lemma whose main idea has been introduced to us by $M$. Dauge [9]. Hereafter the norms in the Lebesgue spaces $L^{p}(\Omega), L^{p}\left(\Omega, \mathbb{R}^{2}\right)$ and $L^{p}(\Omega, \mathbb{C})$ will be denoted by $\|\cdot\|_{L^{p}(\Omega)}$ or $\|\cdot\|_{p}$, and the norms in the Sobolev spaces $W^{k, p}(\Omega, \mathbb{R})$, $W^{k, p}\left(\Omega, \mathbb{R}^{2}\right)$ and $W^{k, p}(\Omega, \mathbb{C})$ will be denoted by $\|\cdot\|_{W^{k, p}(\Omega)}$ or $\|\cdot\|_{k, p}$.

Lemma 2.1. Let $S_{\alpha}$ denote an infinite sector of angle $\alpha \in(0, \pi]$, i.e.,

$$
S_{\alpha}=\left\{(x, y) \in \mathbb{R}^{2}: 0<\arg (x+i y)<\alpha\right\} .
$$

Let further

$$
\mathcal{H}_{\alpha}=\left\{u \in H^{1}\left(S_{\alpha}\right): u(r \cos \alpha, r \sin \alpha)=0, \forall r>0\right\},
$$

and

$$
\Theta_{\alpha}^{D N}=\inf _{\substack{u \in \mathcal{H}_{\alpha} \\\|u\|_{2}=1}} \int_{S_{\alpha}}|(\nabla-i F) u|^{2} d x,
$$

where $F$ is a magnetic potential satisfying $\operatorname{curl} F=1$ in $S_{\alpha}$. Then,

$$
\Theta_{\alpha}^{D N}=\Theta_{0}
$$

where

$$
\Theta_{0}=\inf _{\substack{u \in H^{1}\left(S_{\pi}\right) \\\|u\|_{2}=1}} \int_{S_{\pi}}|(\nabla-i F) u|^{2} d x .
$$

Proof. Let $0<\alpha_{1}<\alpha_{2} \leq \pi$ and $u \in \mathcal{H}_{\alpha_{1}}$. Let further $\tilde{u} \equiv u$ in $S_{\alpha_{1}}$ and $\tilde{u} \equiv 0$ in $S_{\alpha_{2}} \backslash S_{\alpha_{1}}$. Clearly $\tilde{u} \in \mathcal{H}_{\alpha_{2}}$, and hence it follows that $\Theta_{\alpha_{1}}^{D N} \geq \Theta_{\alpha_{2}}^{D N}$. Consequently,

$$
\Theta_{\alpha}^{D N} \geq \Theta_{\pi}^{D N}, \quad \forall \alpha \leq \pi
$$

From the definition of $\Theta_{0}$ it follows, however, that $\Theta_{\pi}^{D N} \geq \Theta_{0}$, and hence, $\Theta_{\alpha}^{D N} \geq \Theta_{0}$ for all $\alpha \in(0, \pi]$. The proof of (2.1) now easily follows from the proof of Persson's Theorem [11, Appendix B] providing the upper bound $\Theta_{0}$ for the essential spectrum of the magnetic Dirichlet-Neumann Laplacian in $S_{\alpha}$. 
Let $\mathcal{D} \subset \Omega$ have a smooth boundary, except at the corners of $\partial \Omega$. As in [3] we let

$$
\mu_{\epsilon}(\mathcal{A}, \mathcal{D})=\inf _{\substack{u \in \mathcal{H} \\\|\mathcal{H}\|_{2}=1}} \int_{\mathcal{D}}|\epsilon \nabla-i \mathcal{A} u|^{2} d x
$$

wherein

$$
\mathcal{H}(\mathcal{D})=\left\{u \in H^{1}(\mathcal{D}): u=0 \text { on } \partial \mathcal{D} \backslash\left(\partial \mathcal{D} \cap \partial \Omega_{i}\right)\right\} .
$$

Let

$$
\mathcal{S}=\overline{\partial \Omega_{c}} \cap \overline{\partial \Omega_{i}} \cap \overline{\partial \mathcal{D}}
$$

denote the corners of $\Omega$ belonging to $\partial \mathcal{D}$. Following [7] we set for a given magnetic potential $\mathcal{A} \in C^{1}\left(\bar{\Omega}, \mathbb{R}^{2}\right)$,

$$
b=\inf _{x \in \mathcal{D}}|\operatorname{curl} \mathcal{A}| \quad ; \quad b^{\prime}=\inf _{x \in \partial \mathcal{D} \cap \partial \Omega_{i}}|\operatorname{curl} \mathcal{A}| .
$$

The following proposition is similar to a result in [7] obtained for a Neumann boundary condition. Here we treat a Dirichlet-Neumann boundary condition and allow, in addition, some dependence of the magnetic potential on the semi-classical parameter.

Proposition 2.2. Let $a \in W^{1, \infty}\left(\mathcal{D}, \mathbb{R}^{2}\right)$. There exist $C>0$ and $\epsilon_{0}>0$ such that for all $0<\epsilon<\epsilon_{0}$ we have

$$
\mu_{\epsilon}\left(\mathcal{A}+\epsilon^{1 / 2} a, \mathcal{D}\right) \geq \epsilon \min \left(b, \Theta_{0} b^{\prime}\right)\left[1-C\left(1+\|\nabla a\|_{\infty}^{2}\right) \epsilon^{1 / 3}\right] .
$$

Proof. The case $b=0$ being trivial, we assume that $b>0$. We begin by introducing for any $\epsilon>0$ a partition of unity (cf. also [15]), i.e., families $\left\{\eta_{i}\right\}_{i=1}^{K} \subset C^{\infty}(\Omega)$, and $\left\{x_{i}\right\}_{i=1}^{K} \subset \mathcal{D}$ satisfying

$$
\sum_{i=1}^{K} \eta_{i}^{2}=1 \quad ; \quad \operatorname{supp} \eta_{i} \subset B\left(x_{i}, \epsilon^{1 / 3}\right) \quad ; \quad \sum_{i=1}^{K}\left|\nabla \eta_{i}\right|^{2} \leq \frac{C}{\epsilon^{2 / 3}},
$$

where $C>0$ is independent of $\epsilon$.

It can be easily verified that for any $u \in H^{1}(\Omega, \mathbb{C})$

$\left\|\left(\epsilon \nabla-i\left[\mathcal{A}+\epsilon^{1 / 2} a\right]\right) u\right\|_{2}^{2}=\sum_{i=1}^{K}\left[\left\|\left(\epsilon \nabla-i\left[\mathcal{A}+\epsilon^{1 / 2} a\right]\right)\left(\eta_{i} u\right)\right\|_{2}^{2}-\epsilon^{2}\left\|u \nabla \eta_{i}\right\|_{2}^{2}\right]$. 
We now set

$$
v_{i}=\eta_{i} u \exp \left(-i \epsilon^{-1 / 2} a\left(x_{i}\right) \cdot x\right)
$$

to obtain that

$$
\begin{aligned}
& \left\|\left(\epsilon \nabla-i\left[\mathcal{A}+\epsilon^{1 / 2} a\right]\right)\left(\eta_{i} u\right)\right\|_{2}^{2} \\
= & \left\|\left(\epsilon \nabla-i\left[\mathcal{A}+\epsilon^{1 / 2}\left(a-a\left(x_{i}\right)\right)\right]\right) v_{i}\right\|_{2}^{2} \\
\geq & \left.\left(1-\epsilon^{1 / 3}\right)\left\|(\epsilon \nabla-i \mathcal{A}) v_{i}\right\|_{2}^{2}-\epsilon^{2 / 3} \| a-a\left(x_{i}\right)\right) v_{i} \|_{2}^{2} \\
\geq & \left(1-\epsilon^{1 / 3}\right) \mu_{\epsilon}(\mathcal{A}, \mathcal{D})\left\|v_{i}\right\|_{2}^{2}-C \epsilon^{4 / 3}\left\|v_{i}\right\|_{2}^{2} .
\end{aligned}
$$

Substituting the above into (2.5) yields

$$
\left\|\left(\epsilon \nabla-i\left[\mathcal{A}+\epsilon^{1 / 2} a\right]\right) u\right\|_{2}^{2} \geq\left(1-\epsilon^{1 / 3}\right) \mu_{\epsilon}(\mathcal{A}, \mathcal{D})\|u\|_{2}^{2}-C \epsilon^{4 / 3}\left(1+\|\nabla a\|_{\infty}^{2}\right)\|u\|_{2}^{2} .
$$

By following the same steps of the proof of Theorem 7.1 in [7] we can establish that

$$
\mu_{\epsilon}(\mathcal{A}, \mathcal{D}) \geq \epsilon \min \left(b, \Theta_{0} b^{\prime}, \Theta_{\alpha}^{D N} \inf _{x \in \mathcal{S}}|\operatorname{curl} \mathcal{A}|\right)\left(1-C \epsilon^{1 / 2}\right) .
$$

The lemma now follows from (2.1), (2.6), and (2.7).

\section{$3 \quad$ Steady-State Solutions}

We begin by considering steady-state solutions of (1.1) $\left(\psi_{k}, A_{\kappa}, \phi_{\kappa}\right) \in$ $H^{1}\left(\Omega, \mathbb{C} \times \mathbb{R}^{2} \times \mathbb{R}\right)$ in the limit $\kappa \rightarrow+\infty$. Hence, we look at the system 
[3, Section 5]

$$
\begin{array}{lr}
-\nabla_{\kappa A_{\kappa}}^{2} \psi_{\kappa}+i \kappa \phi_{\kappa} \psi_{\kappa}=\kappa^{2}\left(1-\left|\psi_{\kappa}\right|^{2}\right) \psi_{\kappa} & \text { in } \Omega, \\
-\operatorname{curl}^{2} A_{\kappa}+\frac{1}{c} \nabla \phi_{\kappa}=\frac{1}{\kappa} \operatorname{Im}\left(\bar{\psi}_{\kappa} \nabla_{\kappa A_{\kappa}} \psi_{\kappa}\right) & \text { in } \Omega, \\
\psi_{\kappa}=0 & \text { on } \partial \Omega_{c}, \\
\nabla_{\kappa A_{\kappa}} \psi_{\kappa} \cdot \nu=0 & \text { on } \partial \Omega_{i}, \\
\frac{\partial \phi_{\kappa}}{\partial \nu}=-c \kappa J(x) & \text { on } \partial \Omega_{c}, \\
\frac{\partial \phi_{\kappa}}{\partial \nu}=0 & \text { on } \partial \Omega_{i}, \\
f_{\partial \Omega} \operatorname{curl} A_{\kappa} d s=\kappa h_{e x}, &
\end{array}
$$

with the additional gauge restriction (1.9). In the above $\left(\psi_{k}, A_{k}, \phi_{k}\right)$ is the same as $(\psi, A, \phi)$ in (1.1). The subscript $\kappa$ has been added to emphasize the limit we consider here. We assume in addition (1.3)-(1.15). By the strong maximum principle we easily obtain that

$$
\left\|\psi_{\kappa}\right\|_{\infty}<1 .
$$

Let $h$ be given by (1.13). It has been demonstrated in [3] that for some $h_{c}>0$, when $h<h_{c} \kappa$, the normal state looses its stability. Since we consider cases for which $1<h \ll \kappa$ it is reasonable to expect that other steady-state solutions would exist. We note, however, that in contrast with the case $J=0$, where the existence of steady-state solutions can be proved using variational arguments (inapplicable in our case), existence of steady-state solutions to (3.1) is yet an open problem when an electric current is applied. We shall address time-dependent solutions in the next section.

Next we set

$$
A_{1, \kappa}=A_{\kappa}-\kappa A_{n}, \quad \phi_{1, \kappa}=\phi_{\kappa}-c \kappa \phi_{n} .
$$

Set further

$$
B_{\kappa}=\operatorname{curl} A_{\kappa}, \quad B_{1, \kappa}=\operatorname{curl} A_{1, \kappa} .
$$


By (3.1b) we then have

$$
\begin{cases}\operatorname{curl} B_{1, \kappa}+\frac{1}{c} \nabla \phi_{1, \kappa}=\frac{1}{\kappa} \operatorname{Im}\left(\bar{\psi}_{\kappa} \nabla_{\kappa A_{\kappa}} \psi_{\kappa}\right) & \text { in } \Omega, \\ \frac{\partial \phi_{1, \kappa}}{\partial \nu}=0 & \text { on } \partial \Omega, \\ f_{\partial \Omega} B_{1, \kappa}(x) d s=0 . & \end{cases}
$$

Note that since $\partial B_{1, \kappa} / \partial \tau=\partial \phi_{1, \kappa} / \partial \nu=0$ on $\partial \Omega$ we must have by (3.5c) that

$$
\left.B_{1, \kappa}\right|_{\partial \Omega} \equiv 0 .
$$

Taking the divergence of (3.1b) yields, with the aid of the imaginary part of (3.1a), that $\phi_{\kappa}$ is a weak solution of

$$
\begin{cases}-\Delta \phi_{\kappa}+c\left|\psi_{\kappa}\right|^{2} \phi_{\kappa}=0 & \text { in } \Omega, \\ \frac{\partial \phi_{\kappa}}{\partial \nu}=c \kappa J & \text { on } \partial \Omega_{c}, \\ \frac{\partial \phi_{\kappa}}{\partial \nu}=0 & \text { on } \partial \Omega_{i} .\end{cases}
$$

By assumption $\phi_{\kappa} \in H^{1}(\Omega)$ and hence, by [3, Proposition A.2] we obtain that $\phi_{\kappa} \in W^{2, p}(\Omega)$ for all $p \geq 2$, hence $\phi_{\kappa} \in C^{1}(\bar{\Omega})$. By (3.3b) we then have

$$
\begin{cases}-\Delta \phi_{1, \kappa}+c\left|\psi_{\kappa}\right|^{2} \phi_{1, \kappa}=-\kappa c^{2}\left|\psi_{k}\right|^{2} \phi_{n} & \text { in } \Omega, \\ \frac{\partial \phi_{1, \kappa}}{\partial \nu}=0 & \text { on } \partial \Omega .\end{cases}
$$

Let $K=\left\|\phi_{n}\right\|_{\infty}$ and $w=\phi_{1, \kappa}+K \kappa c$. Clearly,

$$
\begin{cases}-\Delta w+c\left|\psi_{\kappa}\right|^{2} w=-\kappa c^{2}\left|\psi_{k}\right|^{2}\left(\phi_{n}-K\right) \geq 0 & \text { in } \Omega, \\ \frac{\partial w}{\partial \nu}=0 & \text { on } \partial \Omega .\end{cases}
$$

It can be easily verified that $w$ is the minimizer in $H^{1}(\Omega)$ of

$$
\mathcal{J}(v)=\|\nabla v\|_{2}^{2}+c\left\|\psi_{\kappa} v\right\|_{2}^{2}+\kappa c^{2}\left\langle\left|\psi_{k}\right|^{2}\left(\phi_{n}-K\right), v\right\rangle .
$$

As

$$
\mathcal{J}\left(v_{+}\right) \leq \mathcal{J}(v),
$$

it easily follows that $w \geq 0$, that is

$$
\phi_{1, \kappa}+K \kappa c \geq 0 .
$$


In a similar manner we obtain

$$
\phi_{1, \kappa}-K \kappa c \leq 0,
$$

which together with (3.3b) yields

$$
\left\|\phi_{\kappa}\right\|_{\infty} \leq C(\Omega, J) c \kappa
$$

We now apply again Proposition A.2 in [3] to obtain that for any $p \geq 2$

$$
\left\|\phi_{\kappa}\right\|_{2, p} \leq C(\Omega, J) c \kappa .
$$

We note that all elliptic estimates must be taken with special care since $\Omega$ possesses corners. The necessary details (with references therein) can be found in Appendices A and B of [3] .

Next we set for $\delta>0$ and $\kappa \geq 1$,

$$
D_{\delta}(\kappa)=\left\{x \in \Omega:\left|B_{\kappa}(x)\right|>(1+\delta) \kappa\right\},
$$

and

$$
S_{\delta}=\left\{x \in \Omega:\left|B_{n}(x)\right|>(1+\delta)\right\} .
$$

By either (1.15a) or (1.15b), it follows that for $0<\delta<h-1, S_{\delta} \neq \emptyset$. Below we show that the same is true for $D_{\delta}(\kappa)$. Note that (1.12) implies that $S_{\delta}$ consists of two disjoint sets:

$$
S_{\delta}=S_{\delta, 1} \cup S_{\delta, 2},
$$

one near $\partial \Omega_{i, 1}$ denoted by $S_{\delta, 1}$, and one near $\partial \Omega_{i, 2}$ denoted by $S_{\delta, 2}$. We then let

$$
\mathcal{C}_{\delta, j}=\partial S_{\delta, j} \backslash\left(\partial \Omega \cap \partial S_{\delta, j}\right), \quad j=1,2,
$$

and

$$
\mathcal{C}_{\delta}=\mathcal{C}_{\delta, 1} \cup \mathcal{C}_{\delta, 2}
$$

We can now state and prove

Lemma 3.1. For any $0<\alpha<1$ there exists $\kappa_{0}=\kappa_{0}(\Omega, J, \alpha)>0$ such that for all $\kappa \geq \kappa_{0}$ and $0<\delta<h-1$, we have

$$
S_{\delta+\kappa^{-\alpha}} \subset D_{\delta}(\kappa)
$$


Proof. Step 1: Prove that for some $C(\Omega, J)>0$

$$
\left\|A_{\kappa}\right\|_{\infty} \leq C \kappa .
$$

Taking the divergence of (3.5a) yields, with the aid of (3.5b),

$$
\begin{cases}-\Delta \phi_{1, \kappa}=-\frac{c}{\kappa} \operatorname{div} \operatorname{Im}\left(\bar{\psi}_{\kappa} \nabla_{\kappa A_{\kappa}} \psi_{\kappa}\right) & \text { in } \Omega, \\ \frac{\partial \phi_{1, \kappa}}{\partial \nu}=0 & \text { on } \partial \Omega .\end{cases}
$$

Multiplying the above equation by $\phi_{1, \kappa}$ and integrating by parts then yields, with the aid of $(3.2)$ and $(3.1 \mathrm{c}, \mathrm{d})$,

$$
\left\|\nabla \phi_{1, \kappa}\right\|_{2} \leq \frac{c}{\kappa}\left\|\nabla_{\kappa A_{\kappa}} \psi_{\kappa}\right\|_{2} .
$$

Taking the inner product of (3.1a) with $\psi_{\kappa}$ yields, after integration by parts

$$
\left\|\nabla_{\kappa A_{\kappa}} \psi_{\kappa}\right\|_{2}^{2}=\kappa^{2}\left\|\psi_{\kappa}\right\|_{2}^{2} .
$$

By (3.17) we then obtain that

$$
\left\|\nabla \phi_{1, \kappa}\right\|_{2} \leq C c .
$$

Since curl $B_{1, \kappa}=\nabla_{\perp} B_{1, \kappa}$, the boundedness of $\left\|\nabla B_{1, \kappa}\right\|_{2}$ then easily follows from the above and (3.5). Consequently,

$$
\frac{1}{c}\left\|\nabla \phi_{1, \kappa}\right\|_{2}+\left\|\nabla B_{1, \kappa}\right\|_{2} \leq C .
$$

Note that $\nabla \phi_{1, \kappa}$ and $\nabla_{\perp} B_{1, \kappa}$ are respectively the $L^{2}$ projections of $\operatorname{Im}\left(\bar{\psi}_{\kappa} \nabla_{\kappa A_{\kappa}} \psi_{\kappa}\right)$ on

$$
H_{0}^{0}(\operatorname{curl}, \Omega)=\left\{\widehat{V} \in L^{2}\left(\Omega, \mathbb{R}^{2}\right): \operatorname{curl} \widehat{V}=0\right\},
$$

and

$$
\mathcal{H}_{d}^{0}:=\left\{\widehat{W} \in L^{2}\left(\Omega, \mathbb{R}^{2}\right): \operatorname{div} \widehat{W}=0 \text { and } \widehat{W} \cdot \nu=0 \text { on } \partial \Omega\right\} .
$$

Next, we attempt to estimate $\left\|\nabla \phi_{1, \kappa}\right\|_{p}$ and $\left\|\nabla B_{1, \kappa}\right\|_{p}$ for any $p>2$. Since $\Omega$ is simply-connected, we may conclude from (1.9), (3.4a) 
and Remark B.2 in [3] that there exists for any $p>2$ a constant $C(p, \Omega)>0$ such that

$$
\left\|A_{\kappa}\right\|_{1, p} \leq C\left\|B_{\kappa}\right\|_{p},
$$

for all $\kappa \geq 1$. Sobolev embeddings then imply

$$
\left\|A_{\kappa}\right\|_{\infty} \leq C\left\|B_{\kappa}\right\|_{p} .
$$

Since $\left\|\nabla B_{1, \kappa}\right\|_{2}$ is uniformly bounded for all $\kappa \geq 1$, we obtain from (3.6), the Poincaré inequality, and Sobolev embeddings that, for any $p>2$ there exists a constant $C(p, \Omega)>0$ such that we have

$$
\left\|B_{1, \kappa}\right\|_{p} \leq C(p, \Omega) .
$$

Hence, recalling from (1.19) that $B_{n} \in L^{p}$ and independent of $c$ and $\kappa$, as $J$ is independent of $\kappa$, there exists a constant $C>0$ such that

$$
\left\|B_{\kappa}\right\|_{p}=\left\|B_{1, \kappa}+\kappa B_{n}\right\|_{p} \leq C \kappa .
$$

Combining the above computations with (3.20) then yields (3.15).

Step 2: Prove (3.14).

We first rewrite $(3.1 \mathrm{a}, \mathrm{c}, \mathrm{d})$ in the following form

$$
\left\{\begin{array}{lc}
\Delta \psi_{\kappa}=2 i \kappa A_{\kappa} \cdot \nabla_{\kappa A_{\kappa}} \psi_{\kappa}+\left|\kappa A_{\kappa}\right|^{2} \psi_{\kappa}-\kappa^{2} \psi_{\kappa}\left(1-\left|\psi_{\kappa}\right|^{2}\right)+i \kappa \phi_{\kappa} \psi_{\kappa} & \text { in } \Omega, \\
\psi_{\kappa}=0 & \text { on } \partial \Omega_{c}, \\
\frac{\partial \psi_{\kappa}}{\partial \nu}=i \kappa\left(A_{\kappa} \cdot \nu\right) \psi_{\kappa}=0 & \text { on } \partial \Omega_{i},
\end{array}\right.
$$

where the last equality follows from (1.9). By (3.15), (3.8), the fact $\left\|\psi_{\kappa}\right\|_{\infty} \leq 1$, Proposition A.3 and Remark A.4 in [3] (note that $\psi_{\kappa}$ vanishes at the corners) we obtain that for some $C(\Omega, p, J)$

$$
\left\|\psi_{\kappa}\right\|_{2, p} \leq C\left[\kappa^{4}+\kappa^{2}\left\|\nabla_{\kappa A_{\kappa}} \psi_{\kappa}\right\|_{p}\right], \quad \forall p>2 .
$$

Sobolev embedding and (3.15) then yield

$$
\left\|\nabla_{\kappa A_{\kappa}} \psi_{\kappa}\right\|_{\infty} \leq C\left[\kappa^{4}+\kappa^{2}\left\|\nabla_{\kappa A_{\kappa}} \psi_{\kappa}\right\|_{p}\right] .
$$

We now use a standard interpolation theorem to obtain that

$$
\left\|\nabla_{\kappa A_{\kappa}} \psi_{\kappa}\right\|_{p} \leq\left\|\nabla_{\kappa A_{\kappa}} \psi_{\kappa}\right\|_{2}^{2 / p}\left\|\nabla_{\kappa A_{\kappa}} \psi_{\kappa}\right\|_{\infty}^{1-2 / p} .
$$


Substituting (3.23) in conjunction with (3.18) into the above inequality then yields

$$
\left\|\nabla_{\kappa A_{\kappa}} \psi_{\kappa}\right\|_{p} \leq C\left[\kappa^{4}+\kappa^{2}\left\|\nabla_{\kappa A_{\kappa}} \psi_{\kappa}\right\|_{p}\right]^{1-2 / p} \kappa^{2 / p} .
$$

Suppose first that

$$
\kappa^{2}<\left\|\nabla_{\kappa A_{\kappa}} \psi_{\kappa}\right\|_{p}
$$

Then, we have

$$
\left\|\nabla_{\kappa A_{\kappa}} \psi_{\kappa}\right\|_{p} \leq C\left\|\nabla_{\kappa A_{\kappa}} \psi_{\kappa}\right\|_{p}^{1-2 / p} \kappa^{2(1-1 / p)} .
$$

Hence,

$$
\left\|\nabla_{\kappa A_{\kappa}} \psi_{\kappa}\right\|_{p} \leq C \kappa^{p-1}
$$

Next, assume that

$$
\left\|\nabla_{\kappa A_{\kappa}} \psi_{\kappa}\right\|_{p} \leq \kappa^{2},
$$

to obtain that

$$
\left\|\nabla_{\kappa A_{\kappa}} \psi_{\kappa}\right\|_{p} \leq C \kappa^{4-6 / p} .
$$

From the above, together with (3.25) we easily conclude that, for any $2<p \leq 3$, there exists a constant $C$ such that

$$
\frac{1}{\kappa}\left\|\nabla_{\kappa A_{\kappa}} \psi_{\kappa}\right\|_{p} \leq C \kappa^{3(1-2 / p)} .
$$

To continue, we need a $W^{1, p}$ estimates for the solution of $\Delta u=f$ where $f \in W^{-1, p}$. We thus apply [13, Theorem 7.1], which is valid for any domain which is bilipschitz equivalent to the unit cube, to (3.16). This yields that, for some $C(p, \Omega)>0$, we have

$$
\left\|\nabla \phi_{1, \kappa}\right\|_{p} \leq C \frac{c}{\kappa}\left\|\nabla_{\kappa A_{\kappa}} \psi_{\kappa}\right\|_{p}, \quad \forall \kappa \geq 1,2<p \leq 3 .
$$

From (3.26) and (3.5) we then obtain that

$$
\frac{1}{c}\left\|\nabla \phi_{1, \kappa}\right\|_{p}+\left\|\nabla B_{1, \kappa}\right\|_{p} \leq C \kappa^{3(p-2) / p} .
$$

Upon (3.28) and (3.6) we use the Poincaré inequality together with Sobolev embeddings to conclude that

$$
\left\|B_{1, \kappa}\right\|_{\infty} \leq C \kappa^{3(p-2) / p}, \quad \forall \kappa>1,2<p \leq 3 .
$$


Let $x \in S_{\delta+\kappa^{-\alpha}}$, namely

$$
\left|B_{n}(x)\right|>\left(1+\delta+\kappa^{-\alpha}\right) .
$$

From (3.29), for some $C(p, \Omega, J)>0$ we have that

$$
\begin{aligned}
\left|B_{\kappa}(x)\right| & >\kappa\left|B_{n}(x)\right|-\left|B_{1, \kappa}(x)\right| \geq\left(1+\delta+\kappa^{-\alpha}\right) \kappa-C \kappa^{3(p-2) / p} \\
& =(1+\delta) \kappa+\left[\kappa^{1-\alpha}-C \kappa^{3(p-2) / p}\right] .
\end{aligned}
$$

By choosing

$$
2<p<\min \left(3, \frac{6}{2+\alpha}\right)
$$

we have $\kappa^{1-\alpha}-C \kappa^{3(p-2) / p}>0$ for sufficiently large $\kappa$. Thus

$$
\left|B_{\kappa}(x)\right|>(1+\delta) \kappa,
$$

and hence $x \in D_{\delta}(\kappa)$. Consequently, $S_{\delta+\kappa^{-\alpha}} \subset D_{\delta}(\kappa)$.

As a byproduct of the proof, we also obtain

Proposition 3.2. For any $2<p \leq 3$, there exists $\kappa_{0} \geq 1$ and $C>0$ such that

$$
\left\|A_{1, \kappa}\right\|_{2, p} \leq C \kappa^{3(p-2) / p}, \quad \forall \kappa \geq \kappa_{0} .
$$

Proof. The proof follows immediately from (3.5), (3.28), and Proposition B.3 in [3].

We can now prove the following semi-classical Agmon estimate for $\psi_{\kappa}$, establishing that it must be exponentially small in $S_{\delta}$.

Proposition 3.3. Suppose that h satisfies (1.15b). Let then $j \in\{1,2\}$ be such that $h_{j}>1 / \Theta_{0}$. There exist $C>0$ and $\delta_{0}>0$, such that, for any $0<\delta \leq \delta_{0}$, some $\kappa_{0}(\delta)$ can be found, for which

$$
\kappa \geq \kappa_{0}(\delta) \Rightarrow \int_{S_{\delta, j}} \exp \left(\delta^{1 / 2} \kappa d\left(x, \mathcal{C}_{\delta, j}\right)\right)\left|\psi_{\kappa}\right|^{2} d x \leq \frac{C}{\delta^{3 / 2}}
$$

where $S_{\delta, j}$ is introduced in (3.11) and $\mathcal{C}_{\delta, j}$ in (3.12). 
Proof. For $\delta>0$, let $\eta \in C^{\infty}(\Omega,[0,1])$ satisfy

$$
\eta(x)= \begin{cases}1 & x \in S_{\delta, j} \\ 0 & x \in \Omega \backslash S_{\delta / 2, j} .\end{cases}
$$

By (1.17) and (1.19), it follows that $\nabla B_{n}$ is bounded and independent of both $\delta$ and $\kappa$. Consequently, there exists a constant $C_{1}>0$ such that

$$
d\left(\mathcal{C}_{\delta, j}, \mathcal{C}_{\delta / 2, j}\right) \geq \frac{\delta}{C_{1}},
$$

and hence, for some $C(\Omega, J)$ and all $0<\delta<\delta_{0}$ we can choose $\eta$ such that

Let further

$$
|\nabla \eta| \leq \frac{C}{\delta} .
$$

$$
\zeta=\chi \eta
$$

where

$$
\chi= \begin{cases}\exp \left(\alpha_{\delta} \kappa d\left(x, \mathcal{C}_{\delta, j}\right)\right) & \text { if } x \in S_{\delta, j}, \\ 1 & \text { if } x \in \Omega \backslash S_{\delta, j} .\end{cases}
$$

We leave the determination of $\alpha_{\delta}$ to a later stage. We further define, for any $r \in\left(0, r_{0}\right), \eta_{r} \in C^{\infty}(\Omega,[0,1])$ and $\tilde{\eta}_{r} \in C^{\infty}(\Omega,[0,1])$ such that

$$
\eta_{r}(x)=\left\{\begin{array}{ll}
1 & d\left(x, \partial \Omega_{i}\right)>r \\
0 & d\left(x, \partial \Omega_{i}\right)<r / 2,
\end{array} \quad \text { and } \quad\left|\nabla \eta_{r}\right|^{2}+\left|\nabla \tilde{\eta}_{r}\right|^{2} \leq \frac{C}{r^{2}},\right.
$$

and

$$
\eta_{r}^{2}+\tilde{\eta}_{r}^{2}=1
$$

Fix $0<\alpha<1$. Multiplying (3.1a) by $\zeta^{2} \bar{\psi}$, integrating by parts yields for the real part

$$
\begin{aligned}
& \left\|\nabla_{\kappa A_{\kappa}}\left(\zeta \tilde{\eta}_{\kappa^{-1 / 2}} \psi_{\kappa}\right)\right\|_{2}^{2}+\left\|\nabla_{\kappa A_{\kappa}}\left(\zeta \eta_{\kappa^{-1 / 2}} \psi_{\kappa}\right)\right\|_{2}^{2} \\
\leq & \kappa^{2}\left\|\zeta \psi_{\kappa}\right\|_{2}^{2}+\left\|\zeta \psi_{\kappa} \nabla \eta_{\kappa^{-1 / 2}}\right\|_{2}^{2}+\left\|\zeta \psi_{\kappa} \nabla \tilde{\eta}_{\kappa^{-1 / 2}}\right\|_{2}^{2}+\left\|\psi_{\kappa} \nabla \zeta\right\|_{2}^{2} .
\end{aligned}
$$

Observing that $\left\langle\psi_{\kappa} \nabla \chi, \psi_{\kappa} \nabla \eta\right\rangle=0$, we obtain

$$
\left\|\psi_{\kappa} \nabla \zeta\right\|_{2}^{2} \leq \alpha_{\delta}^{2} \kappa^{2}\left\|\psi_{\kappa} \zeta\right\|_{2}^{2}+\left\|\psi_{\kappa} \nabla \eta\right\|_{2}^{2} .
$$


Hence,

$$
\begin{aligned}
& \left\|\nabla_{\kappa A_{\kappa}}\left(\zeta \tilde{\eta}_{\kappa^{-1 / 2}} \psi_{\kappa}\right)\right\|_{2}^{2}+\left\|\nabla_{\kappa A_{\kappa}}\left(\zeta \eta_{\kappa^{-1 / 2}} \psi_{\kappa}\right)\right\|_{2}^{2} \\
\leq & \kappa^{2}\left(1+\alpha_{\delta}^{2}+C \kappa^{-1}\right)\left\|\zeta \psi_{\kappa}\right\|_{2}^{2}+\left\|\psi_{\kappa} \nabla \eta\right\|_{2}^{2} .
\end{aligned}
$$

We now use (2.4) and (3.30) to obtain, for sufficiently small $\delta$,

$$
\begin{aligned}
\left\|\nabla_{\kappa A_{\kappa}}\left(\zeta \tilde{\eta}_{\kappa^{-1 / 2}} \psi_{\kappa}\right)\right\|_{2}^{2} & \geq \kappa^{4} \mu_{\kappa^{-2}}\left(A_{n}+\kappa^{-1} A_{1, \kappa}, S_{\delta / 2}\right)\left\|\zeta \tilde{\eta}_{\kappa^{-1 / 2}} \psi_{\kappa}\right\|_{2}^{2} \\
& \geq \kappa^{2} \min \left(\Theta_{0} h_{j}, 1+\delta / 2\right)\left[1-C \kappa^{-2 / 3}\right]\left\|\zeta \tilde{\eta}_{\kappa^{-1 / 2}} \psi_{\kappa}\right\|_{2}^{2} \\
& \geq\left(1+\frac{\delta}{2}\right) \kappa^{2}\left[1-C \kappa^{-2 / 3}\right]\left\|\zeta \tilde{\eta}_{\kappa^{-1 / 2}} \psi_{\kappa}\right\|_{2}^{2} .
\end{aligned}
$$

By [5, Theorem 2.9] we have, since $\zeta \eta_{\kappa^{-1 / 2}} \psi_{\kappa}$ vanishes on $\partial \Omega$,

$$
\left\|\nabla_{\kappa A_{\kappa}}\left(\zeta \eta_{\kappa^{-1 / 2}} \psi_{\kappa}\right)\right\|_{2}^{2} \geq\left(1+\delta / 2-\kappa^{-1 / 2}\right) \kappa^{2}\left\|\zeta \eta_{\kappa^{-1 / 2}} \psi_{\kappa}\right\|_{2}^{2} .
$$

Consequently, by (3.34), (3.35), and (3.36), and by choosing

$$
\alpha_{\delta}^{2}=\frac{\delta}{4}
$$

we obtain, that for $\kappa \geq \kappa(\delta)$, with $\kappa(\delta)$ sufficiently large:

$$
\kappa^{2} \frac{\delta}{8}\left\|\zeta \psi_{\kappa}\right\|_{2}^{2} \leq \kappa^{2}\left(\frac{\delta}{4}-\widehat{C} \kappa^{-\frac{1}{2}}\right)\left\|\zeta \psi_{\kappa}\right\|_{2}^{2} \leq\left\|\psi_{\kappa} \nabla \eta\right\|_{2}^{2},
$$

from which (3.31) easily follows.

Next we consider currents satisfying only (1.15a). Let, for $j=1,2$,

$$
\omega_{\delta, j}=\left\{x \in \Omega:(-1)^{j} B_{n}(x)>1+\delta ; d\left(x, \partial \Omega_{i}\right)>\delta\right\},
$$

and

$$
\Gamma_{\delta, j}=\partial \omega_{\delta, j} \backslash \partial \Omega_{c} \cap \partial \omega_{\delta, j}
$$

We can now state

Proposition 3.4. Suppose that for some $j \in\{1,2\}$ we have

$$
1<\left|h_{j}\right| \text {. }
$$

Then, there exist $C>0, \delta_{0}>0$, such that for any $0<\delta<\delta_{0}$, some $\kappa_{0}(\delta)>0$ can be found, for which

$$
\kappa \geq \kappa_{0}(\delta) \Rightarrow \int_{\omega_{\delta, j}} \exp \left(\delta^{1 / 2} \kappa d\left(x, \Gamma_{\delta, j}\right)\right)\left|\psi_{\kappa}\right|^{2} d x \leq \frac{C}{\delta^{3 / 2}} .
$$


Proof. Without loss of generality we may assume $h_{j}>0$; otherwise we apply to (3.1) the transformation $\left(\psi_{\kappa}, A_{\kappa}, \phi_{\kappa}\right) \rightarrow\left(\bar{\psi}_{\kappa},-A_{\kappa},-\phi_{\kappa}\right)$. Let

$$
\check{\chi}= \begin{cases}\exp \left(\frac{1}{2} \delta^{1 / 2} \kappa d\left(x, \Gamma_{\delta, j}\right)\right) & \text { if } x \in \omega_{\delta, j}, \\ 1 & \text { if } x \in \Omega \backslash \omega_{\delta, j} .\end{cases}
$$

Let further $\eta$ and $\eta_{r}$ be given by (3.32) and (3.33) respectively. Then set

$$
\check{\zeta}=\eta_{\delta} \eta \check{\chi} .
$$

The proof proceeds in the same manner as in the previous proposition with $\zeta$ replaced by $\check{\zeta}$ with the difference that now $\check{\zeta} \psi_{\kappa}(x)$ vanishes for all $x \in \partial \Omega_{i}$. Consequently, (1.15a) is no longer necessary (see (3.36)). We use $(1.15 \mathrm{~b})$ to establish that $\omega_{\delta, j}$ is not empty.

We conclude this section by showing that for $\mathcal{O}(\kappa)$ currents (i.e. when $J$ is independent of $\kappa)\left\|\psi_{\kappa}\right\|_{2}$ must be small. To this end we define $\Phi_{n}$ as the solution of $(1.18 \mathrm{a}, \mathrm{b})$, and

$$
\int_{\Omega}\left|\psi_{\kappa}\right|^{2} \Phi_{n} d x=0 .
$$

The above condition is a natural choice as by (3.7) we have that

$$
\int_{\Omega}\left|\psi_{\kappa}\right|^{2} \phi_{\kappa} d x=0 .
$$

It can be easily verified from (2.2) that

$$
\Phi_{n}=\phi_{n}+C(\kappa, c),
$$

where $\phi_{n}$ denotes the solution of (1.18). The constant can be extracted from (3.43):

$$
C(\kappa, c)=-\frac{\int_{\Omega} \phi_{n}\left|\psi_{\kappa}\right|^{2} d x}{\int_{\Omega}\left|\psi_{\kappa}\right|^{2} d x},
$$

from which we get the following upper bound (independent of $\kappa$ and $c$ )

$$
|C(\kappa, c)| \leq\left\|\phi_{n}\right\|_{\infty}<+\infty .
$$


Proposition 3.5. Under Assumptions (1.3)-(1.15) there exists $C(J, \Omega)>0$ and $\kappa_{0}>0$ such that for any $\kappa \geq \kappa_{0}$,

$$
\left\|\psi_{\kappa}\right\|_{2} \leq C(J, \Omega)\left(1+c^{-1 / 2}\right)^{1 / 3} \kappa^{-1 / 6} .
$$

Proof. Let $\Phi_{1, \kappa}=\phi_{k}-c \kappa \Phi_{n}$. An immediate consequence of (3.7) is that

$$
\begin{cases}-\Delta \Phi_{1, \kappa}+c|\psi|_{\kappa}^{2} \Phi_{1, \kappa}=-c^{2}|\psi|_{\kappa}^{2} \kappa \Phi_{n} & \text { in } \Omega, \\ \frac{\partial \Phi_{1, \kappa}}{\partial \nu}=0 & \text { on } \partial \Omega .\end{cases}
$$

Taking the inner product with $\Phi_{n}$ yields, with the aid of (3.43),

$$
\begin{aligned}
& \kappa\left\|\psi_{\kappa} \Phi_{n}\right\|_{2}^{2}=-\frac{1}{c^{2}}\left\langle\nabla \Phi_{n}, \nabla \Phi_{1, \kappa}\right\rangle+\frac{1}{c}\left\langle\left\langle\left|\psi_{\kappa}\right| \Phi_{n},\left|\psi_{\kappa}\right|\left(\Phi_{1, \kappa}-\left(\Phi_{1, \kappa}\right)_{\Omega}\right\rangle\right.\right. \\
\leq & \frac{1}{c^{2}}\left\|\nabla \Phi_{n}\right\|_{2}\left\|\nabla \Phi_{1, \kappa}\right\|_{2}+\frac{1}{c}\left\|\psi_{\kappa} \Phi_{n}\right\|_{2}\left\|\psi_{\kappa}\left(\Phi_{1, \kappa}-\left(\Phi_{1, \kappa}\right)_{\Omega}\right)\right\|_{2},
\end{aligned}
$$

where $\left(\Phi_{1, \kappa}\right)_{\Omega}$ is the average of $\Phi_{1, \kappa}$ in $\Omega$.

With the aid of (3.19) (note that $\nabla \phi_{1, \kappa}=\nabla \Phi_{1, \kappa}$ ), the fact that $\left|\psi_{\kappa}\right| \leq 1$, and the Poincaré inequality we then obtain

$$
\left\|\psi_{\kappa} \Phi_{n}\right\|_{2} \leq C \kappa^{-1 / 2}\left(1+c^{-1 / 2}\right) .
$$

We now set

$$
\mathcal{U}_{\kappa}=\left\{x \in \Omega:\left|\Phi_{n}(x)\right|<\left(1+c^{-1 / 2}\right)^{2 / 3} \kappa^{-1 / 3}\right\} .
$$

By (3.43) the level set $\Phi_{n}^{-1}(0)$ lies inside $\Omega$. Let $x_{0} \in \Phi_{n}^{-1}(\tau)$ for some $\tau \neq 0$, and set

$$
\Gamma_{\perp}=B_{n}^{-1}\left(B_{n}\left(x_{0}\right)\right) .
$$

By (1.16a) $B_{n}$ is the conjugate harmonic function of $\Phi_{n}$, and hence $\Gamma_{\perp}$ must be perpendicular to $\Phi_{n}^{-1}(\tau)$ at $x_{0}$. Note that in $[3,(2.3)]$ we showed that $B_{n}^{-1}(\mu)$ is a simple smooth curve connecting the two connected components of $\partial \Omega_{c}$ for any $h_{1}<\mu<h_{2}$. We denote by $\tilde{\Gamma}_{\perp}$ the subcurve of $\Gamma_{\perp}$ originating from $x_{0}$ in the direction where $\Phi_{n}$ decreases if $\tau>0$ or increases if $\tau<0$, and terminating either on $\Phi_{n}^{-1}(0)$ or on the boundary. Clearly,

$$
\left|\tilde{\Gamma}_{\perp}\right| \inf _{x \in \Omega}\left|\nabla \Phi_{n}\right| \leq\left|\int_{\tilde{\Gamma}_{\perp}} \nabla \Phi_{n} d s\right| \leq|\tau|,
$$


where $\int_{\Gamma} \vec{V}$ denotes the circulation of $\vec{V}$ along the path $\Gamma$. In $[3, \S 2.3]$ we have established that $\left|\nabla \Phi_{n}\right|=\left|\nabla B_{n}\right|>0$ in $\bar{\Omega}$. It follows that

$$
d\left(x_{0}, \partial \Omega \cup \Phi_{n}^{-1}(0)\right) \leq\left|\tilde{\Gamma}_{\perp}\right| \leq C|\tau| .
$$

Let

$$
\tilde{\mathcal{U}}_{\kappa}(r)=\left\{x \in \Omega \mid d\left(x, \partial \Omega \cup \Phi_{n}^{-1}(0)\right) \leq r\left(1+c^{-1 / 2}\right)^{2 / 3} \kappa^{-1 / 3}\right\} .
$$

By (3.50) we obtain that for sufficiently large $r$ there exists $\kappa_{0}(r)$ such that for all $\kappa>\kappa_{0}$ and $c \in \mathbb{R}$ we have $\mathcal{U}_{\kappa} \subseteq \tilde{\mathcal{U}}_{\kappa}(r)$. Consequently,

$$
\left|\mathcal{U}_{\kappa}\right| \leq C\left(1+c^{-1 / 2}\right)^{2 / 3} \kappa^{-1 / 3}\left(\left|\Phi_{n}^{-1}(0)\right|+|\partial \Omega|\right) \leq C\left(1+c^{-1 / 2}\right)^{2 / 3} \kappa^{-1 / 3} .
$$

By (3.49) we have that

$$
\left\|\psi_{\kappa}\right\|_{L^{2}\left(\Omega \backslash \mathcal{U}_{\kappa}\right)} \leq C\left(1+c^{-1 / 2}\right)^{1 / 3} \kappa^{-1 / 6}
$$

whereas from (3.51) and (3.2) we learn that

$$
\left\|\psi_{\kappa}\right\|_{L^{2}\left(\mathcal{U}_{\kappa}\right)} \leq C\left(1+c^{-1 / 2}\right)^{1 / 3} \kappa^{-1 / 6} .
$$

The proposition can now be readily verified.

An immediate conclusion is that whenever $c \kappa \gg 1,\left|\psi_{\kappa}\right|$ is small. If $c=\mathcal{O}\left(\kappa^{-1}\right),\left|\psi_{\kappa}\right|$ may not tend to 0 as $\kappa \rightarrow \infty$. Further research is necessary to establish this point.

Remark 3.6. If, for some $0<\alpha<1$, we assume that $J=J(\cdot, \kappa)$ satisfies

$$
\|J\| \leq C \kappa^{-\alpha}
$$

then (3.48) and (3.19) remain valid. Assuming $c=1$, and using this time (3.28), we obtain instead of (3.49), for any $0<\beta$, that

$$
\left\|\psi_{\kappa} \Phi_{n}\right\|_{2} \leq C_{\beta} \kappa^{-1 / 2}\left(\kappa^{\alpha / 2}+\kappa^{\beta}\left\|\psi_{\kappa}\right\|_{2}^{1 / 2}\right)
$$

Using the above, with sufficiently small $\beta$, we obtain, similarly to the derivation of (3.46)

$$
\left\|\psi_{\kappa}\right\|_{2} \leq C \kappa^{-(1-\alpha) / 6}
$$


which implies

$$
\left\|\psi_{\kappa}\right\|_{2} \underset{\kappa \rightarrow+\infty}{\longrightarrow} 0
$$

This result stands in sharp contrast with the behavior obtained in the absence of electric potential [22, 4].

\section{Time-Dependent Analysis}

In this section we return to the time-dependent problem as introduced in (1.1). For convenience we set here

$$
c=1 \text {. }
$$

$$
\begin{array}{lr}
\frac{\partial \psi_{\kappa}}{\partial t}-\nabla_{\kappa A_{\kappa}}^{2} \psi_{\kappa}+i \kappa \phi_{\kappa} \psi_{\kappa}=\kappa^{2}\left(1-\left|\psi_{\kappa}\right|^{2}\right) \psi_{\kappa} & \text { in }(0,+\infty) \times \Omega, \\
\frac{\partial A_{\kappa}}{\partial t}+\nabla \phi_{\kappa}+\operatorname{curl}^{2} A_{\kappa}=\frac{1}{\kappa} \operatorname{Im}\left(\bar{\psi}_{\kappa} \nabla_{\kappa A_{\kappa}} \psi_{\kappa}\right) & \text { in }(0,+\infty) \times \Omega, \\
\psi=0 & \text { on }(0,+\infty) \times \partial \Omega_{c}, \\
\nabla_{\kappa A_{\kappa}} \psi_{\kappa} \cdot \nu=0 & \text { on }(0,+\infty) \times \partial \Omega_{i}, \\
\frac{\partial \phi_{\kappa}}{\partial \nu}=-\kappa J(x) & \text { on }(0,+\infty) \times \partial \Omega_{c}, \\
\frac{\partial \phi_{\kappa}}{\partial \nu}=0 & \text { on }(0,+\infty) \times \partial \Omega_{i}, \\
f_{\partial \Omega} \operatorname{curl} A_{\kappa} d s=\kappa h_{e x} & \text { on }(0,+\infty), \\
\psi(0, x)=\psi_{0}(x) & \text { in } \Omega, \\
A(0, x)=A_{0}(x) & \text { in } \Omega .
\end{array}
$$

We assume again (1.2)-(1.10), (1.12), and (1.15). Since in the time dependent case $\phi_{\kappa}$ is determined up to a constant in view of (1.8) and (1.9), we can further impose

$$
\int_{\Omega} \phi_{\kappa}(t, x) d x=0, \quad \forall t>0
$$


It follows from (1.2) by the maximum principle (see [3, Theorem 2.6]) that

$$
\left\|\psi_{\kappa}(t, \cdot)\right\|_{\infty} \leq 1, \quad \forall t \geq 0 .
$$

We recall from [3, Subsection 2.4] the following spectral entity

$$
\lambda=\inf _{\substack{V \in \mathcal{H}_{d} \\\|V\|_{2}=1}}\|\operatorname{curl} V\|_{2}^{2},
$$

where

$$
\mathcal{H}_{d}=\left\{V \in H^{1}\left(\Omega, \mathbb{R}^{2}\right): \operatorname{div} V=0,\left.V\right|_{\partial \Omega} \cdot \nu=0\right\} .
$$

We further recall from [3, Proposition 2.5] that, under condition $\left(R_{1}\right)$ on $\partial \Omega$,

$$
\lambda=\lambda^{D}:=\inf _{\substack{u \in H_{0}^{1}(\Omega) \\\|u\|_{2}=1}}\|\nabla u\|_{2}^{2}>0 .
$$

We retain our definition of the normal fields $\left(A_{n}, \phi_{n}\right)$ via (1.16). For the solution $\left(A_{\kappa}, \phi_{\kappa}\right)$ of (4.1) we set

$$
\begin{aligned}
& A_{1, \kappa}(t, x)=A_{\kappa}(t, x)-\kappa A_{n}(x), \\
& \phi_{1, \kappa}(t, x)=\phi_{\kappa}(t, x)-\kappa \phi_{n}(x), \\
& B_{\kappa}(t, x)=\operatorname{curl} A_{\kappa}(t, x), \\
& B_{1, \kappa}(t, x)=\operatorname{curl} A_{1, \kappa}(t, x) .
\end{aligned}
$$

Clearly,

$$
\begin{array}{rlrl}
\frac{\partial A_{1, \kappa}}{\partial t}+\nabla \phi_{1, \kappa}+\operatorname{curl} B_{1, \kappa} & =\frac{1}{\kappa} \operatorname{Im}\left(\bar{\psi}_{\kappa} \nabla_{\kappa A_{\kappa}} \psi_{\kappa}\right) & & \text { in }(0,+\infty) \times \Omega, \\
\frac{\partial \phi_{1, \kappa}}{\partial \nu} & =0 & \text { on }(0,+\infty) \times \partial \Omega, \\
f_{\partial \Omega} B_{1, \kappa}(t, x) d s & =0 & \text { in }(0,+\infty) .
\end{array}
$$

We begin by the following auxiliary estimate. We recall that $\|A(t, \cdot)\|_{1,2}=\|A(t, \cdot)\|_{H^{1}\left(\Omega, \mathbb{R}^{2}\right)}$. 
Lemma 4.1. Let $A_{1, \kappa}$ and $B_{1, \kappa}$ be defined by (4.5). Suppose that $\left\|A_{1, \kappa}(\cdot, 0)\right\|_{2} \leq M$ (where $M$ may depend on $\kappa$ ). Then, under the above assumptions, there exists $t^{*}(M)$ and a constant $C=C\left(\Omega, t^{*}\right)>0$ such that for all $t>t^{*}$ and $\kappa \geq 1$ we have

$$
\left\|A_{1, \kappa}(t, \cdot)\right\|_{1,2}+\left\|A_{1, \kappa}\right\|_{L^{2}\left(t, t+1, H^{2}(\Omega)\right)} \leq C .
$$

Proof. By [3, Lemma 5.3] there exists a constant $C=C(\Omega)>0$ such that for sufficiently large $\kappa$

$$
\left\|A_{1, \kappa}(t, \cdot)\right\|_{2}^{2} \leq\left(\left\|A_{1, \kappa}(0, \cdot)\right\|_{2}^{2}+\frac{C}{\kappa^{2}}\right) e^{-\lambda t}+C \int_{0}^{t} e^{-\lambda(t-\tau)}\left\|\psi_{\kappa}(\tau, \cdot)\right\|_{2}^{2} d \tau .
$$

From (4.3), we then get

$$
\left\|A_{1, \kappa}(t, \cdot)\right\|_{2}^{2} \leq\left(\left\|A_{1, \kappa}(0, \cdot)\right\|_{2}^{2}+\frac{C}{\kappa^{2}}\right) e^{-\lambda t}+\frac{C}{\lambda}
$$

We thus have

$$
\left\|A_{1, \kappa}(t, \cdot)\right\|_{2}^{2} \leq(M+C) e^{-\lambda t}+\frac{C}{\lambda}
$$

Hence, there exists $t_{0}^{*}(M)$, such that for $t \geq t_{0}^{*}(M)$, we have

$$
\left\|A_{1, \kappa}(t, \cdot)\right\|_{2} \leq \frac{2 C}{\lambda} .
$$

Next, we apply [3, Theorem C.1 (Formula C.4)] to the operator $\mathcal{L}^{(1)}$ (as introduced there in Example (4) above this theorem) to obtain that

$$
\begin{aligned}
& \left\|A_{1, \kappa}\right\|_{L^{\infty}\left(t_{0}, t_{0}+1, H^{1}(\Omega)\right)}+\left\|A_{1, \kappa}\right\|_{L^{2}\left(t_{0}, t_{0}+1, H^{2}(\Omega)\right)} \\
& \quad \leq \frac{C}{\kappa}\left\|\operatorname{Im}\left\{\bar{\psi}_{\kappa} \nabla_{\kappa A_{\kappa}} \psi_{\kappa}\right\}\right\|_{L^{2}\left(t_{0}, t_{0}+1, L^{2}(\Omega)\right)}+C\left\|A_{1, \kappa}\left(t_{0}, \cdot\right)\right\|_{1,2} .
\end{aligned}
$$

with a constant $C$ independent of $t_{0}$. Since from (4.1a) (cf. [3]) we can easily get that

$$
\left\|\nabla_{\kappa A_{\kappa}} \psi_{\kappa}(t, \cdot)\right\|_{2}^{2} \leq \kappa^{2}\left\|\psi_{\kappa}(t, \cdot)\right\|_{2}^{2}-\frac{1}{2} \frac{d\left\|\psi_{\kappa}(t, \cdot)\right\|_{2}^{2}}{d t},
$$

we obtain by integrating over $\left(t_{0}, t_{0}+1\right)$

$$
\left\|\nabla_{\kappa A_{\kappa}} \psi_{\kappa}\right\|_{L^{2}\left(t_{0}, t_{0}+1, L^{2}(\Omega)\right)}^{2} \leq \kappa^{2}\left\|\psi_{\kappa}\right\|_{L^{2}\left(t_{0}, t_{0}+1, L^{2}(\Omega)\right)}^{2}+\frac{1}{2}\left\|\psi_{\kappa}\left(t_{0}, \cdot\right)\right\|_{2}^{2},
$$


and note for later reference that it implies

$$
\left\|\nabla_{\kappa A_{\kappa}} \psi_{\kappa}\right\|_{L^{2}\left(t_{0}, t_{0}+1, L^{2}(\Omega)\right)} \leq C(\Omega) \kappa
$$

Implementing the upper bound (4.12) in (4.10), yields

$$
\begin{aligned}
& \left\|A_{1, \kappa}\right\|_{L^{\infty}\left(t_{0}, t_{0}+1, H^{1}(\Omega)\right)}+\left\|A_{1, \kappa}\right\|_{L^{2}\left(t_{0}, t_{0}+1, H^{2}(\Omega)\right)} \\
\leq & C\left[1+\left\|\psi_{\kappa}\right\|_{L^{2}\left(t_{0}, t_{0}+1, L^{2}(\Omega)\right)}+\frac{1}{\kappa}\left\|\psi_{\kappa}\left(t_{0}, \cdot\right)\right\|_{2}+\left\|A_{1, \kappa}\left(t_{0}, \cdot\right)\right\|_{1,2}\right] .
\end{aligned}
$$

We next apply [3, Theorem C.1 (Formula C.2)] to obtain in precisely the same manner

$$
\begin{aligned}
& \left\|A_{1, \kappa}\right\|_{L^{\infty}\left(t_{0}, t_{0}+1, H^{1}(\Omega)\right)} \\
& \quad \leq C\left[1+\left\|\psi_{\kappa}\right\|_{L^{2}\left(t_{0}-1, t_{0}+1, L^{2}(\Omega)\right)}+\frac{1}{\kappa}\left\|\psi_{\kappa}\left(t_{0}, \cdot\right)\right\|_{2}+\left\|A_{1, \kappa}\left(t_{0}-1, \cdot\right)\right\|_{2}\right] .
\end{aligned}
$$

The above together with (4.3) and (4.9) yields, for $t_{0} \geq t_{0}^{*}+1$,

$$
\left\|A_{1, \kappa}\right\|_{L^{\infty}\left(t_{0}, t_{0}+1, H^{1}(\Omega)\right)}+\left\|A_{1, \kappa}\right\|_{L^{2}\left(t_{0}, t_{0}+1, H^{2}(\Omega)\right)} \leq C,
$$

which implies (4.7), with $t^{*}=t_{0}^{*}(M)+1$.

Remark 4.2. Since our interest is in the limit as $t \rightarrow+\infty$, Lemma 4.1 allows us to assume in the sequel, without any loss of generality, that (4.7) is satisfied for all $t \geq 0$. We have just to make a translation $t \mapsto t-t^{*}$ and to observe that $\psi_{\kappa}\left(t^{*}, \cdot\right)$ has the same properties as $\psi_{0}$.

Proposition 4.3. Let $\omega_{\delta, j}(j \in\{1,2\})$ be defined in (3.37). Suppose that for some $j \in\{1,2\}$ we have that

$$
1<\left|h_{j}\right|
$$

Then, there exist $C>0$ and $\delta_{0}>0$, and, for any $0<\delta<\delta_{0}, \kappa_{0}(\delta) \geq 1$ such that, for $\kappa \geq \kappa_{0}(\delta)$,

$$
\limsup _{t \rightarrow \infty} \int_{\omega_{\delta, j}}\left|\psi_{\kappa}\right|^{2}(t, x) d x \leq \frac{C_{\delta}}{\kappa^{2}} .
$$


Proof. Without loss of generality we may assume $h_{j}>0$; otherwise we apply to (4.1) the transformation $\left(\psi_{\kappa}, A_{\kappa}, \phi_{\kappa}\right) \rightarrow\left(\bar{\psi}_{\kappa},-A_{\kappa},-\phi_{\kappa}\right)$.

Step 1: Let, for $n \geq 1$,

$$
a_{n}=\left\|\widehat{\zeta} \psi_{\kappa}\right\|_{L^{\infty}\left(n-1, n, L^{2}(\Omega)\right)} .
$$

Prove that for all $\delta \in(0,1)$ and $\kappa \geq \kappa_{0}(\delta)$,

$$
a_{n}^{2} \leq C \delta^{-3}\left(\kappa^{-2}+\kappa^{-1}\left(a_{n}+a_{n-1}\right)\right) .
$$

Let $\eta$ and $\eta_{r}$ be given by (3.32) and (3.33) respectively. Then, set

$$
\widehat{\zeta}=\eta \eta_{\delta}
$$

Multiplying (4.1a) by $\widehat{\zeta}^{2} \bar{\psi}_{\kappa}$ and integrating by parts yields

$\frac{1}{2} \frac{d}{d t}\left(\left\|\widehat{\zeta} \psi_{\kappa}(t, \cdot)\right\|_{2}^{2}\right)+\left\|\nabla_{\kappa A_{\kappa}}\left(\widehat{\zeta} \psi_{\kappa}(t, \cdot)\right)\right\|_{2}^{2} \leq \kappa^{2}\left\|\widehat{\zeta} \psi_{\kappa}(t, \cdot)\right\|_{2}^{2}+\left\|\psi_{\kappa}(t, \cdot) \nabla \widehat{\zeta}\right\|_{2}^{2}$.

By [5, Theorem 2.9], we have

$$
\begin{aligned}
\left\|\nabla_{\kappa A_{\kappa}}\left(\widehat{\zeta} \psi_{\kappa}(t, \cdot)\right)\right\|_{2}^{2} & \geq\left\langle\kappa B_{\kappa}(t, \cdot) \widehat{\zeta} \psi_{\kappa}(t, \cdot), \widehat{\zeta} \psi_{\kappa}(t, \cdot)\right\rangle \\
& =\kappa^{2}\left\langle B_{n} \widehat{\zeta} \psi_{\kappa}(t, \cdot), \widehat{\zeta} \psi_{\kappa}(t, \cdot)\right\rangle+\left\langle\kappa B_{1, \kappa}(t, \cdot) \widehat{\zeta} \psi_{\kappa}(t, \cdot), \widehat{\zeta} \psi_{\kappa}(t, \cdot)\right\rangle \\
& \geq \kappa^{2}\left(1+\frac{\delta}{2}\right)\left\|\widehat{\zeta} \psi_{\kappa}(t, \cdot)\right\|_{2}^{2}+\left\langle\kappa B_{1, \kappa} \widehat{\zeta} \psi_{\kappa}(t, \cdot), \widehat{\zeta} \psi_{\kappa}(t, \cdot)\right\rangle .
\end{aligned}
$$

We can thus write

$$
\begin{aligned}
& \frac{1}{2} \frac{d}{d t}\left(\left\|\widehat{\zeta} \psi_{\kappa}(t, \cdot)\right\|_{2}^{2}\right)+\frac{\kappa^{2} \delta}{2}\left\|\widehat{\zeta} \psi_{\kappa}(t, \cdot)\right\|_{2}^{2} \\
& \quad \leq\left\|\psi_{\kappa}(t, \cdot) \nabla \eta\right\|_{2}^{2}+\left\|\psi_{\kappa}(t, \cdot) \nabla \eta_{\delta}\right\|_{2}^{2}-\left\langle\kappa B_{1, \kappa}(t, \cdot) \widehat{\zeta} \psi_{\kappa}(t, \cdot), \widehat{\zeta} \psi_{\kappa}(t, \cdot)\right\rangle .
\end{aligned}
$$

Since

$$
\left\|\psi_{\kappa}(t, \cdot) \nabla \eta\right\|_{2}^{2}+\left\|\psi_{\kappa}(t, \cdot) \nabla \eta_{\delta}\right\|_{2}^{2} \leq \frac{C}{\delta^{2}},
$$

we obtain that

$$
\begin{aligned}
& \frac{1}{2} \frac{d}{d t}\left(\left\|\widehat{\zeta} \psi_{\kappa}(t, \cdot)\right\|_{2}^{2}\right)+\frac{\kappa^{2} \delta}{2}\left\|\widehat{\zeta} \psi_{\kappa}(t, \cdot)\right\|_{2}^{2} \\
& \quad \leq \frac{C}{\delta^{2}}-\left\langle\kappa B_{1, \kappa}(t, \cdot) \widehat{\zeta} \psi_{\kappa}(t, \cdot), \widehat{\zeta} \psi_{\kappa}(t, \cdot)\right\rangle .
\end{aligned}
$$


From (4.19) we can conclude that

$$
\begin{aligned}
\left\|\widehat{\zeta} \psi_{\kappa}(t, \cdot)\right\|_{2}^{2} \leq & \left\|\widehat{\zeta} \psi_{0}\right\|_{2}^{2} e^{-\delta \kappa^{2} t}+\frac{C}{\delta^{3} \kappa^{2}} \\
& +2 \int_{0}^{t} e^{-\delta \kappa^{2}(t-\tau)}\left|\left\langle\kappa B_{1, \kappa}(\tau, \cdot) \widehat{\zeta} \psi_{\kappa}(\tau, \cdot), \widehat{\zeta} \psi_{\kappa}(\tau, \cdot)\right\rangle\right| d \tau .
\end{aligned}
$$

To estimate the last term on the right-hand-side of (4.20), we start from

$$
\begin{aligned}
& \int_{0}^{t} e^{-\delta \kappa^{2}(t-\tau)}\left|\left\langle\kappa B_{1, \kappa}(\tau, \cdot) \widehat{\zeta} \psi_{\kappa}(\tau, \cdot), \widehat{\zeta} \psi_{\kappa}(\tau, \cdot)\right\rangle\right| d \tau \\
\leq & \kappa\left[\int_{0}^{t} e^{-\delta \kappa^{2}(t-\tau)}\left\|B_{1, \kappa}(\tau, \cdot)\right\|_{2}^{2} d \tau \cdot \int_{0}^{t} e^{-\delta \kappa^{2}(t-\tau)}\left\|\widehat{\zeta} \psi_{\kappa}(\tau, \cdot)\right\|_{4}^{4} d \tau\right]^{1 / 2} .
\end{aligned}
$$

With Remark 4.2 in mind, we use (4.3) to obtain

$$
\int_{0}^{t} e^{-\delta \kappa^{2}(t-\tau)}\left\|B_{1, \kappa}(\tau, \cdot)\right\|_{2}^{2} d \tau \leq \frac{C}{\delta \kappa^{2}} .
$$

Implementing the above estimate, we obtain

$$
\begin{gathered}
\int_{0}^{t} e^{-\delta \kappa^{2}(t-\tau)}\left|\left\langle\kappa B_{1, \kappa}(\tau, \cdot) \widehat{\zeta} \psi_{\kappa}(\tau, \cdot), \widehat{\zeta} \psi_{\kappa}(\tau, \cdot)\right\rangle\right| d \tau \\
\leq C \delta^{-\frac{1}{2}}\left[\int_{0}^{t} e^{-\delta \kappa^{2}(t-\tau)}\left\|\widehat{\zeta} \psi_{\kappa}(\tau, \cdot)\right\|_{4}^{4} d \tau\right]^{1 / 2} .
\end{gathered}
$$

To control of the right hand side we now write for $t \geq 1$

$$
\begin{aligned}
& {\left[\int_{0}^{t} e^{-\delta \kappa^{2}(t-\tau)}\left\|\widehat{\zeta} \psi_{\kappa}(\tau, \cdot)\right\|_{4}^{4} d \tau\right]^{1 / 2}} \\
& \leq\left[\int_{0}^{t-1} e^{-\delta \kappa^{2}(t-\tau)}\left\|\widehat{\zeta} \psi_{\kappa}(\tau, \cdot)\right\|_{4}^{4} d \tau\right]^{1 / 2}+\left[\int_{t-1}^{t} e^{-\delta \kappa^{2}(t-\tau)}\left\|\widehat{\zeta} \psi_{\kappa}(\tau, \cdot)\right\|_{4}^{4} d \tau\right]^{1 / 2} \\
& \leq C\left[\int_{t-1}^{t} e^{-\delta \kappa^{2}(t-\tau)}\left\|\widehat{\zeta} \psi_{\kappa}(\tau, \cdot)\right\|_{2}^{2} d \tau\right]^{1 / 2}+C \delta^{-\frac{1}{2}} \kappa^{-1} e^{-\frac{\delta}{2} \kappa^{2}} \\
& \leq C \delta^{-\frac{1}{2}} \kappa^{-1}\left\|\widehat{\zeta} \psi_{\kappa}\right\|_{L^{\infty}\left(t-1, t, L^{2}(\Omega)\right)}+C \delta^{-\frac{1}{2}} \kappa^{-1} e^{-\frac{\delta}{2} \kappa^{2}} .
\end{aligned}
$$

Substituting the above into (4.20) yields, with a new constant $C$, for $\kappa$ large enough, and for $t \geq 1$,

$$
\begin{aligned}
& \left\|\widehat{\zeta} \psi_{\kappa}(t, \cdot)\right\|_{2}^{2} \\
& \quad \leq C \delta^{-3} \kappa^{-2}+C \delta^{-1} \kappa^{-1} e^{-\frac{\delta}{2} \kappa^{2}}+C \delta^{-1} \kappa^{-1}\left\|\widehat{\zeta} \psi_{\kappa}\right\|_{L^{\infty}\left(t-1, t, L^{2}(\Omega)\right)} .
\end{aligned}
$$


From which we easily obtain (4.17).

Step 2: Prove (4.16).

By (4.3) we have

$$
0<a_{n} \leq C
$$

which readily yields

$$
a_{n} \leq C \delta^{-\frac{3}{2}} \kappa^{-\frac{1}{2}}
$$

We improve the above estimate by reimplementing (4.17). To this end we set

$$
\widehat{C}:=C \delta^{-\frac{3}{2}},
$$

and then let

$$
a_{n}=\frac{\widehat{C}}{\kappa} \alpha_{n} .
$$

Substituting into (4.17) yields

$$
\alpha_{n}^{2} \leq 1+\alpha_{n-1}+\alpha_{n} .
$$

Suppose that for some $N \geq 0$, we have $\alpha_{N} \leq 1+\sqrt{2}$, then $\alpha_{N+1} \leq 1+\sqrt{2}$ and hence $\alpha_{n} \leq 1+\sqrt{2}$ for all $n \geq N$.

If $\alpha_{n-1}>1+\sqrt{2}$ for any $n$, we have, with $\hat{\alpha}_{n}=\alpha_{n}-\frac{1}{2}$,

$$
\hat{\alpha}_{n}^{2} \leq \frac{7}{4}+\hat{\alpha}_{n-1}<\hat{\alpha}_{n-1}^{2} .
$$

Hence, $\hat{\alpha}_{n}<\hat{\alpha}_{n-1}$ which means that $\hat{\alpha}_{n}$ converges as a positive decreasing sequence, and necessarily to a limit smaller than $1 / 2+\sqrt{2}$. We thus conclude that

$$
\lim \sup \alpha_{n} \leq 1+\sqrt{2}
$$

and hence

$$
\lim \sup a_{n} \leq \frac{C(1+\sqrt{2})}{\delta^{3 / 2} \kappa},
$$

from which (4.16) can easily be deduced.

We next obtain the following improvement over (4.13) for $\nabla_{\kappa A_{\kappa}}\left(\widehat{\zeta} \psi_{\kappa}\right)$. 
Proposition 4.4. Let $p \geq 2$. For any $\delta>0$, there exists $\kappa_{0}(\delta)$ and $C(\delta)$ such that for $\kappa \geq \kappa(\delta)$ we have, with $u=\widehat{\zeta} \psi_{\kappa}$, the following estimate:

$$
\left\|\nabla_{\kappa A_{\kappa}} u\right\|_{L^{p}\left(t_{0}, t_{0}+1 ; L^{p}(\Omega)\right)} \leq C(\delta) \kappa^{6(1-2 / p)} .
$$

Proof. Step 1: Prove that for some $C(\delta)>0$ we have, for sufficiently large $\kappa$ that

$$
\|u\|_{L^{2}\left(t_{0}-1, t_{0}+1, H^{2}(\Omega)\right)} \leq C \kappa^{3} .
$$

We rewrite $(4.1 \mathrm{a}, \mathrm{c}, \mathrm{d})$ in the form

$$
\begin{cases}\frac{\partial \psi_{\kappa}}{\partial t}-\Delta \psi_{\kappa}=-2 i \kappa A_{\kappa} \cdot \nabla \psi_{\kappa}-\left|\kappa A_{\kappa}\right|^{2} \psi_{\kappa}+\kappa^{2} \psi_{\kappa}\left(1-\left|\psi_{\kappa}\right|^{2}\right) & \\ -i \kappa \phi_{\kappa} \psi_{\kappa} & \text { in }(0,+\infty) \times \Omega, \\ \psi_{\kappa}=0 & \text { on }(0,+\infty) \times \partial \Omega_{c}, \\ \frac{\partial \psi_{\kappa}}{\partial \nu}=i \kappa A_{\kappa} \psi_{\kappa} \cdot \nu=0 & \text { on }(0,+\infty) \times \partial \Omega_{i} .\end{cases}
$$

Clearly, by our choice of $\widehat{\zeta}$,

$$
\left\{\begin{array}{lll}
\frac{\partial u}{\partial t}-\Delta u=\widehat{\zeta}\left[-2 i \kappa A_{\kappa} \cdot \nabla \psi_{\kappa}-\left|\kappa A_{\kappa}\right|^{2} \psi_{\kappa}+\kappa^{2} \psi_{\kappa}\left(1-\left|\psi_{\kappa}\right|^{2}\right)\right. & \\
& \left.-i \kappa \phi_{\kappa} \psi_{\kappa}\right]+2 \nabla \widehat{\zeta} \cdot \nabla \psi_{\kappa}+\psi_{\kappa} \Delta \widehat{\zeta} & \text { in }(0,+\infty) \times \Omega, \\
u=0 & \text { on }(0,+\infty) \times \partial \Omega .
\end{array}\right.
$$

By [3, Theorem C.1] (this time applied to the Dirichlet Laplacian in $\Omega$ ) in the interval $\left(t_{0}-1, t_{0}+1\right)$

$$
\begin{aligned}
& \|u\|_{L^{2}\left(t_{0}, t_{0}+1, H^{2}(\Omega)\right)} \\
\leq & \left\|\widehat{\zeta}\left[-2 i \kappa A_{\kappa} \cdot \nabla \psi_{\kappa}-\left|\kappa A_{\kappa}\right|^{2} \psi_{\kappa}+\kappa^{2} \psi_{\kappa}\left(1-\left|\psi_{\kappa}\right|^{2}\right)-i \kappa \phi_{\kappa} \psi_{\kappa}\right]\right\|_{L^{2}\left(t_{0}-1, t_{0}+1, L^{2}\left(\Omega, \mathbb{R}^{2}\right)\right)} \\
& +\left\|2 \nabla \widehat{\zeta} \cdot \nabla \psi_{\kappa}+\psi_{\kappa} \Delta \widehat{\zeta}\right\|_{L^{2}\left(t_{0}-1, t_{0}+1, L^{2}\left(\Omega, \mathbb{R}^{2}\right)\right)}+C\left\|u\left(t_{0}-1, \cdot\right)\right\|_{2} .
\end{aligned}
$$$$
\text { By (4.3) we have that }
$$$$
\left\|2 \nabla \widehat{\zeta} \cdot \nabla \psi_{\kappa}+\psi_{\kappa} \Delta \widehat{\zeta}\right\|_{L^{2}\left(t_{0}-1, t_{0}+1, L^{2}(\Omega)\right)} \leq C\left(1+\left\|\nabla \psi_{\kappa}\right\|_{L^{2}\left(t_{0}-1, t_{0}+1, L^{2}(\Omega)\right)}\right) .
$$

As

$$
\left\|\nabla \psi_{\kappa}\right\|_{L^{2}\left(t_{0}-1, t_{0}+1, L^{2}(\Omega)\right)} \leq\left\|\nabla_{\kappa A_{\kappa}} \psi_{\kappa}\right\|_{L^{2}\left(t_{0}-1, t_{0}+1, L^{2}(\Omega)\right)}+\left\|\kappa A_{\kappa} \psi_{\kappa}\right\|_{L^{2}\left(t_{0}-1, t_{0}+1, L^{2}(\Omega)\right)},
$$


we obtain in view of (4.12) and (4.7) that

$$
\left\|\nabla \psi_{\kappa}\right\|_{L^{2}\left(t_{0}-1, t_{0}+1, L^{2}(\Omega)\right)} \leq C \kappa^{2} .
$$

Substituting the above into (4.27) yields

$$
\left\|2 \nabla \widehat{\zeta} \cdot \nabla \psi_{\kappa}+\psi_{\kappa} \Delta \widehat{\zeta}\right\|_{L^{2}\left(t_{0}-1, t_{0}+1, L^{2}(\Omega)\right)} \leq C \kappa^{2}
$$

We next observe that

$\left\|\widehat{\zeta}\left|\kappa A_{\kappa}\right|^{2} \psi_{\kappa}\right\|_{L^{2}\left(t_{0}-1, t_{0}+1, L^{2}(\Omega)\right)} \leq \kappa^{2}\left\|A_{\kappa}\right\|_{L^{4}\left(t_{0}-1, t_{0}+1, L^{\infty}\left(\Omega, \mathbb{R}^{2}\right)\right)}^{2}\left\|\widehat{\zeta} \psi_{\kappa}\right\|_{L^{2}\left(t_{0}-1, t_{0}+1, L^{2}(\Omega)\right)}$

Since $\widehat{\zeta}$ is supported in the set $\omega_{\delta, j}$, we may use (4.16), which together with Agmon's inequality [1, Lemma 13.2], (4.5), and (4.7), yield, for $t_{0}$ large enough,

$$
\begin{aligned}
& \left\|\left.\widehat{\zeta}\left|\kappa A_{\kappa}\right|\right|^{2} \psi_{\kappa}\right\|_{L^{2}\left(t_{0}-1, t_{0}+1, L^{2}(\Omega)\right)} \\
\leq & C \kappa\left\|A_{\kappa}\right\|_{L^{\infty}\left(t_{0}-1, t_{0}+1, L^{2}\left(\Omega, \mathbb{R}^{2}\right)\right)}\left\|A_{\kappa}\right\|_{L^{2}\left(t_{0}-1, t_{0}+1, H^{2}\left(\Omega, \mathbb{R}^{2}\right)\right)} \\
\leq & C \kappa^{3} .
\end{aligned}
$$

Similarly,

$$
\begin{aligned}
& \left\|\widehat{\zeta} \kappa A_{\kappa} \cdot \nabla \psi_{\kappa}\right\|_{L^{2}\left(t_{0}-1, t_{0}+1, L^{2}(\Omega)\right)} \\
\leq & \kappa\left\|A_{\kappa}\right\|_{L^{4}\left(t_{0}, t_{0}+1, L^{\infty}\left(\Omega, \mathbb{R}^{2}\right)\right)}\left[\|u\|_{L^{4}\left(t_{0}-1, t_{0}+1, H^{1}(\Omega)\right)}+\left\|\psi_{\kappa} \nabla \widehat{\zeta}\right\|_{L^{4}\left(t_{0}-1, t_{0}+1, L^{2}(\Omega)\right)}\right] \\
\leq & C \kappa^{2}+C \kappa^{2}\|u\|_{L^{2}\left(t_{0}-1, t_{0}+1, H^{2}(\Omega)\right)}^{1 / 2}\|u\|_{L^{\infty}\left(t_{0}-1, t_{0}+1, L^{2}(\Omega)\right)}^{\frac{1}{2}} \\
\leq & C\left[\kappa^{2}+\kappa^{3 / 2}\|u\|_{L^{2}\left(t_{0}-1, t_{0}+1, H^{2}(\Omega)\right)}^{\frac{1}{2}}\right] .
\end{aligned}
$$

Substituting (4.31) together with (4.29), and (4.30) into (4.26) yields with the aid of (4.3)

$$
\|u\|_{L^{2}\left(t_{0}, t_{0}+1, H^{2}(\Omega)\right)} \leq C\left[\kappa^{3}+\kappa^{3 / 2}\|u\|_{L^{2}\left(t_{0}-1, t_{0}+1, H^{2}(\Omega)\right)}^{\frac{1}{2}}\right]
$$

Proceeding as in the proof of Proposition 4.3, we can assume $C \geq 1$ in (4.32) and set

$$
\alpha_{n}=C^{-1} \kappa^{-\frac{3}{2}}\|u\|_{L^{2}\left(n, n+1, H^{2}(\Omega)\right)}^{\frac{1}{2}} .
$$


We now can rewrite (4.32) in the form

$$
\alpha_{n}^{2} \leq\left(1+\alpha_{n-1}+\alpha_{n}\right),
$$

which is precisely (4.21). We can thus conclude (4.22), and hence, for a new value of $C,(4.25)$ easily follows.

Step 2: Prove that

$$
\left\|\nabla_{\kappa A_{\kappa}} u\right\|_{L^{2}\left(t_{0}, t_{0}+1, H^{1}\left(\Omega, \mathbb{R}^{2}\right)\right)} \leq C \kappa^{3} .
$$

It can be easily verified that

$$
\left\|\nabla_{\kappa A_{\kappa}} u(t, \cdot)\right\|_{1,2} \leq\|u(t, \cdot)\|_{2,2}+\kappa\left\|\left|A_{\kappa}\right| \nabla u(t, \cdot)\right\|_{2}+\kappa\left\|u \nabla A_{\kappa}(t, \cdot)\right\|_{2} .
$$

Furthermore, in the same manner we have obtained (4.31) we obtain, with the aid of (4.25)

$$
\kappa\left\|\left|A_{\kappa}\right| \nabla u\right\|_{L^{2}\left(t_{0}, t_{0}+1, L^{2}\left(\Omega, \mathbb{R}^{2}\right)\right)} \leq C \kappa^{3 / 2}\|u\|_{L^{2}\left(t_{0}, t_{0}+1, H^{2}\left(\Omega, \mathbb{R}^{2}\right)\right)}^{1 / 2} \leq C \kappa^{3} .
$$

By (4.3) and (4.7) we have that

$$
\kappa\left\|u \nabla A_{\kappa}\right\|_{L^{2}\left(t_{0}, t_{0}+1, L^{2}\left(\Omega, \mathbb{R}^{2}\right)\right)} \leq C \kappa^{2} .
$$

We can now conclude (4.33) from (4.34).

Step 3: Prove (4.24).

In $[3,(5.35)]$ it was shown that

$$
\left\|\nabla_{\kappa A_{\kappa}} \psi_{\kappa}\right\|_{L^{\infty}\left(t_{0}, t_{0}+1 ; L^{2}(\Omega)\right)} \leq C \kappa^{3} .
$$

(Note that while the setting in [3] is different then - in particular, we assume there $J \sim \mathcal{O}(\kappa)$ - the estimate is still valid in the present case because $c=1$.) Hence, we get

$$
\left\|\nabla_{\kappa A_{\kappa}} u\right\|_{L^{\infty}\left(t_{0}, t_{0}+1 ; L^{2}(\Omega)\right)} \leq C \kappa^{3} .
$$


We now use Gagliardo-Nirenberg interpolation inequality (see [19]) to obtain

$$
\begin{aligned}
& \left\|\nabla_{\kappa A_{\kappa}} u\right\|_{L^{p}\left(t_{0}, t_{0}+1 ; L^{p}(\Omega)\right)}^{p} \\
& \leq C \int_{t_{0}}^{t_{0}+1}\left\|\nabla_{\kappa A_{\kappa}} u(t, \cdot)\right\|_{1,2}^{p-2}\left\|\nabla_{\kappa A_{\kappa}} u(t, \cdot)\right\|_{2}^{2} d t \\
& \leq C\left\|\nabla_{\kappa A_{\kappa}} u\right\|_{L^{2}\left(t_{0}, t_{0}+1 ; H^{1}(\Omega)\right)}^{p-2}\left\|\nabla_{\kappa A_{\kappa}} u\right\|_{L^{\frac{4}{4-p}}\left(t_{0}, t_{0}+1 ; L^{2}(\Omega)\right)}^{2}
\end{aligned}
$$

Consequently,

$$
\begin{aligned}
&\left\|\nabla_{\kappa A_{\kappa}} u\right\|_{L^{p}\left(t_{0}, t_{0}+1 ; L^{p}(\Omega)\right)}^{p}\left\|\nabla_{\kappa A_{\kappa}} u\right\|_{L^{2}\left(t_{0}, t_{0}+1 ; L^{2}(\Omega)\right)}^{4-p}\left\|\nabla_{\kappa A_{\kappa}} u\right\|_{L^{\infty}\left(t_{0}, t_{0}+1 ; L^{2}(\Omega)\right)}^{p-2} . \\
& \leq C\left\|\nabla_{\kappa A_{\kappa}} u\right\|_{L^{2}\left(t_{0}, t_{0}+1 ; H^{1}(\Omega)\right)}^{p-2}
\end{aligned}
$$

Multiplying (4.1a) by $\widehat{\zeta}^{2} \bar{\psi}_{\kappa}$ and integrating over $\Omega$ we obtain for the real part that

$$
\left\|\nabla_{\kappa A_{\kappa}(t, \cdot)} u(t, \cdot)\right\|_{2}^{2} \leq \kappa^{2}\|u(t, \cdot)\|_{2}^{2}-\frac{1}{2} \frac{d\|u(t, \cdot)\|_{2}^{2}}{d t}+\left\|\psi_{\kappa}(t, \cdot) \nabla \widehat{\zeta}\right\|_{2}^{2},
$$

Integrating over $\left(t_{0}, t_{0}+1\right)$ and using (4.16) we then obtain, for sufficiently large $t_{0}$

$$
\left\|\nabla_{\kappa A_{\kappa}} u\right\|_{L^{2}\left(t_{0}, t_{0}+1 ; L^{2}(\Omega)\right)}^{2} \leq C .
$$

Substituting the above, (4.33), and (4.36), into (4.37) we then obtain (4.24).

We can now obtain the following improved regularity for $B_{1, \kappa}$

Proposition 4.5. Let $2<p \leq 12 / 5$. For $0<\delta<\delta_{0}$, there exists a constant $C=C(\Omega, \delta)>0$ such that for all $t_{0}>1$ and $\kappa>\kappa_{0}(\delta)$ we have

$$
\left\|B_{1, \kappa}\right\|_{L^{p}\left(t_{0}, t_{0}+1, W^{1, p}\left(\omega_{\delta, j}\right)\right)} \leq C .
$$

Proof. Taking the curl of (4.6) yields that $B_{1, \kappa}$ is a weak solution of

$$
\begin{cases}\frac{\partial B_{1, \kappa}}{\partial t}-\Delta B_{1, \kappa}=\frac{1}{\kappa} \operatorname{curl} \operatorname{Im}\left(\bar{\psi}_{\kappa} \nabla_{\kappa A_{\kappa}} \psi_{\kappa}\right) & \text { in }(0,+\infty) \times \Omega \\ B_{1, \kappa}=0 & \text { on }(0,+\infty) \times \partial \Omega .\end{cases}
$$


Let

$$
\mathcal{B}_{\widehat{\zeta}}=\widehat{\zeta} B_{1, \kappa},
$$

where the cutoff function $\widehat{\zeta}$ is defined by (4.18). Let further $\hat{\Omega}(\delta) \subset \Omega$ be smooth and satisfy

$$
\operatorname{supp} \widehat{\zeta} \subset \hat{\Omega}(\delta) .
$$

As for any $V \in H^{1}\left(\Omega, \mathbb{R}^{2}\right)$ we have that

$$
\operatorname{curl} V=\operatorname{div} V_{\perp},
$$

it can be easily verified from (4.39) that

$$
\left.\frac{\partial \mathcal{B}_{\widehat{\zeta}}}{\partial t}-\Delta \mathcal{B}_{\widehat{\zeta}}=\frac{1}{\kappa} \widehat{\zeta} \operatorname{div}\left(\operatorname{Im}\left(\bar{\psi}_{\kappa} \nabla_{\kappa A_{\kappa}} \psi_{\kappa}\right)_{\perp}\right)-2 \operatorname{div}\left(B_{1, \kappa} \nabla \widehat{\zeta}\right)\right)+B_{1, \kappa} \Delta \widehat{\zeta}
$$

Consequently,

$$
\left\{\begin{aligned}
\frac{\partial \mathcal{B}_{\widehat{\zeta}}}{\partial t}-\Delta \mathcal{B}_{\widehat{\zeta}}= & \frac{1}{\kappa} \widehat{\zeta} \operatorname{div}\left(\operatorname{Im}\left(\bar{\psi}_{\kappa} \nabla_{\kappa A_{\kappa}} \psi_{\kappa}\right)_{\perp}\right) & & \\
& -\operatorname{div}\left(2 B_{1, \kappa} \nabla \widehat{\zeta}+\nabla \Delta_{D}^{-1}\left(B_{1, \kappa} \Delta \widehat{\zeta}\right)\right) & & \text { in }\left(t_{0}-1, t_{0}+1\right) \times \hat{\Omega} \\
\mathcal{B}_{\widehat{\zeta}}= & 0 & & \text { on }\left(t_{0}-1, t_{0}+1\right) \times \partial \hat{\Omega} \\
\mathcal{B}_{\widehat{\zeta}}\left(t_{0}-1, \cdot\right) & =\widehat{\zeta} \mathcal{B}_{1, \kappa}\left(t_{0}-1, \cdot\right) & & \text { in } \hat{\Omega} .
\end{aligned}\right.
$$

In the above $\Delta_{D}^{-1}$ denotes the inverse Dirichlet Laplacian in $\hat{\Omega}$.

In order to apply [8, Theorem 1.6] which is devoted to the case of parabolic operators written in divergence form and with zero initial condition we first decompose the solution of (4.40) into two Cauchy-Dirichlet problems. The first of them is:

$$
\left\{\begin{aligned}
\frac{\partial U_{1}}{\partial t}-\Delta U_{1} & =\operatorname{div} f_{1} & & \text { in }\left(t_{0}-1, t_{0}+1\right) \times \hat{\Omega}, \\
U_{1} & =0 & & \text { on }\left(t_{0}-1, t_{0}+1\right) \times \partial \hat{\Omega}, \\
U_{1}\left(t_{0}-1, \cdot\right) & =0 & & \text { in } \hat{\Omega},
\end{aligned}\right.
$$

in which

$$
f_{1}=\frac{1}{\kappa} \widehat{\zeta}\left(\operatorname{Im}\left(\bar{\psi}_{\kappa} \nabla_{\kappa A_{\kappa}} \psi_{\kappa}\right)_{\perp}\right)-2 B_{1, \kappa} \nabla \widehat{\zeta}-\nabla \Delta_{D}^{-1}\left(B_{1, \kappa} \Delta \widehat{\zeta}\right) .
$$

The second one is:

$$
\left\{\begin{aligned}
\frac{\partial U_{2}}{\partial t}-\Delta U_{2} & =F_{2} & & \text { in }\left(t_{0}-1, t_{0}+1\right) \times \hat{\Omega} \\
U_{2} & =0 & & \text { on }\left(t_{0}-1, t_{0}+1\right) \times \partial \hat{\Omega} \\
U_{2}\left(t_{0}-1, \cdot\right) & =\widehat{\zeta} \mathcal{B}_{1, \kappa}\left(t_{0}-1, \cdot\right) & & \text { in } \hat{\Omega}
\end{aligned}\right.
$$


where

$$
F_{2}:=-\frac{1}{\kappa}\left(\operatorname{Im}\left(\bar{\psi}_{\kappa} \nabla \widehat{\zeta} \cdot\left(\nabla_{\kappa A_{\kappa}} \psi_{\kappa}\right)_{\perp}\right)\right) .
$$

By uniqueness of the weak solution, we have

$$
\mathcal{B}_{\widehat{\zeta}}=U_{1}+U_{2} \text { in }\left(t_{0}-1, t_{0}+1\right) \times \hat{\Omega} .
$$

We now separately estimate $U_{1}$ and $U_{2}$ in $L^{p}\left(t_{0}, t_{0}+1, W^{1, p}(\hat{\Omega})\right)$.

Estimate of $U_{1}$

We apply [8, Theorem 1.6] to obtain

$$
\left\|U_{1}\right\|_{L^{p}\left(t_{0}, t_{0}+1, W^{1, p}(\Omega)\right)} \leq C\left\|f_{1}\right\|_{L^{p}\left(t_{0}, t_{0}+1, L^{p}\left(\Omega, \mathbb{R}^{2}\right)\right.} .
$$

It can be easily verified, by the Gagliardo-Nirenberg interpolation inequality [19] that, for all $2<p<4$, there exists a constant $C$ such that, for any $\phi \in L^{2}\left(t_{0}, t_{0}+1, H^{1}(\Omega)\right) \cap L^{\infty}\left(t_{0}, t_{0}+1, L^{2}(\Omega)\right)$, we have

$$
\begin{aligned}
\|\phi\|_{L^{p}\left(t_{0}, t_{0}+1, L^{p}\left(\Omega, \mathbb{R}^{2}\right)\right)}^{p} & \leq C \int_{t_{0}}^{t_{0}+1}\|\phi(\tau, \cdot)\|_{2}^{2}\|\phi(\tau, \cdot)\|_{1,2}^{p-2} d \tau \\
\leq & C\|\phi\|_{L^{\frac{4}{-p}}\left(t_{0}, t_{0}+1, L^{2}\left(\Omega, \mathbb{R}^{2}\right)\right)}^{2}\|\phi\|_{L^{2}\left(t_{0}, t_{0}+1, H^{1}\left(\Omega, \mathbb{R}^{2}\right)\right)}^{p-2} .
\end{aligned}
$$

By (4.47), (4.7), Remark 4.2, and Sobolev embeddings we have:

$$
\left\|\mathcal{B}_{\widehat{\zeta}}\left(t_{0}, \cdot\right)\right\|_{p}+\left\|\mathcal{B}_{1, \kappa} \nabla \widehat{\zeta}\right\|_{L^{p}\left(t_{0}, t_{0}+1, L^{p}(\Omega)\right)}+\left\|\mathcal{B}_{1, \kappa} \Delta \widehat{\zeta}\right\|_{W^{-1, p}\left(t_{0}, t_{0}+1, L^{p}\left(\Omega, \mathbb{R}^{2}\right)\right)} \leq C .
$$

Furthermore, by (4.3) we have that

$$
\begin{aligned}
& \left\|\widehat{\zeta} \operatorname{Im}\left(\bar{\psi}_{\kappa} \nabla_{\kappa A_{\kappa}} \psi_{\kappa}\right)\right\|_{L^{p}\left(t_{0}, t_{0}+1, L^{p}\left(\Omega, \mathbb{R}^{2}\right)\right)} \\
& \quad \leq\left\|\psi_{\kappa} \nabla \widehat{\zeta}\right\|_{L^{p}\left(t_{0}, t_{0}+1, L^{p}\left(\Omega, \mathbb{R}^{2}\right)\right)}+\left\|\nabla_{\kappa A_{\kappa}}\left(\widehat{\zeta} \psi_{\kappa}\right)\right\|_{L^{p}\left(t_{0}, t_{0}+1, L^{p}\left(\Omega, \mathbb{R}^{2}\right)\right)} \\
& \quad \leq\left\|\nabla_{\kappa A_{\kappa}}\left(\widehat{\zeta} \psi_{\kappa}\right)\right\|_{L^{p}\left(t_{0}, t_{0}+1, L^{p}\left(\Omega, \mathbb{R}^{2}\right)\right)}+C .
\end{aligned}
$$

Substituting the above together with (4.48) into (4.46) yields for $t_{0}$ large enough

$$
\left\|U_{1}\right\|_{L^{p}\left(t_{0}, t_{0}+1, W^{1, p}(\Omega)\right)} \leq C\left(1+\frac{1}{\kappa}\left\|\nabla_{\kappa A_{\kappa}}\left(\widehat{\zeta} \psi_{\kappa}\right)\right\|_{L^{p}\left(t_{0}, t_{0}+1, L^{p}\left(\Omega, \mathbb{R}^{2}\right)\right)}\right) .
$$

Substituting (4.24) into (4.49) yields

$$
\left\|U_{1}\right\|_{L^{p}\left(t_{0}, t_{0}+1, W^{1, p}(\Omega)\right)} \leq C\left(1+\kappa^{5-12 / p}\right) \leq \widehat{C},
$$


since $2<p \leq 12 / 5$.

Estimate of $U_{2}$

Here we apply first $L^{2}$ estimates and then combine them with Sobolev's estimates. It is in this part that we need the information on $F_{2}$ and $U_{2}$ in $\left[t_{0}-1, t_{0}+1\right) \times \hat{\Omega}$ in order to bound the various norms on $\left(t_{0}, t_{0}+1\right) \times \hat{\Omega}$. We begin by applying once again [3, Theorem C.1] (combining $(C .1)$ and (C.2) there) to obtain

$$
\begin{aligned}
& \left\|U_{2}\right\|_{L^{2}\left(t_{0}, t_{0}+1, H^{2}\right)}+\left\|U_{2}\right\|_{L^{\infty}\left(t_{0}, t_{0}+1, H^{1}\right)} \\
& \quad \leq C\left(\left\|F_{2}\right\|_{L^{2}\left(\left(t_{0}-1, t_{0}+1\right) \times \hat{\Omega}\right)}+\left\|U_{2}\left(t_{0}-1, \cdot\right)\right\|_{L^{2}(\hat{\Omega})}\right),
\end{aligned}
$$

where $F_{2}$ and $U_{2}\left(t_{0}-1, \cdot\right)$ given in (4.44) and (4.43). Applying GagliardoNirenberg's inequality yields, for $2<p<4$,

$$
\left\|U_{2}\right\|_{L^{p}\left(t_{0}, t_{0}+1, W^{1, p}\right)} \leq C\left(\left\|F_{2}\right\|_{L^{2}\left(\left(t_{0}-1, t_{0}+1\right) \times \hat{\Omega}\right)}+\left\|U_{2}\left(t_{0}-1, \cdot\right)\right\|_{L^{2}(\hat{\Omega})}\right),
$$

By (4.13) we have that

$$
\left\|F_{2}\right\|_{L^{2}\left(\left(t_{0}-1, t_{0}+1\right) \times \hat{\Omega}\right)} \leq C .
$$

Furthermore, using (4.7), with Remark 4.2 in mind yields

$$
\left\|U_{2}\left(t_{0}-1, \cdot\right)\right\|_{2} \leq C .
$$

Consequently, by (4.51), there exist, for any $2<p<4$ and any $\delta>0$, constants $C(\delta)$ and $\kappa(\delta)$ such that for any $\kappa \geq \kappa_{0}(\delta)$ and any $t_{0}>1$ we have

$$
\left\|U_{2}\right\|_{L^{p}\left(t_{0}, t_{0}+1, W^{1, p}\right)} \leq C(\delta) .
$$

The combination of (4.50) and (4.52) together with (4.45) completes the proof of the proposition.

We can now establish the exponential decay of $\psi_{\kappa}$.

Proposition 4.6. Let $\omega_{\delta, j}(j \in\{1,2\})$ be given by (3.37). Suppose that for some $k \in\{1,2\}$ (4.15) is satisfied. Then, there exist $C>0$ and 
$\delta_{0}>0$, and, for any $0<\delta<\delta_{0}, \kappa_{0}(\delta)$, such that for any $\kappa \geq \kappa_{0}(\delta)$ we have

$$
\limsup _{t \rightarrow \infty} \int_{\omega_{\delta, j}} \exp \left(\delta^{1 / 2} \kappa d\left(x, \Gamma_{\delta, j}\right)\right)\left|\psi_{\kappa}\right|^{2}(t, x) d x \leq C .
$$

Proof. Without loss of generality we may, as before, assume $h_{j}>0$. Let $\check{\chi}$ and $\check{\zeta}$ be defined by (3.41) and (3.42).

Step 1: Prove that

$\left\|\check{\zeta} \psi_{\kappa}(t, \cdot)\right\|_{2}^{2} \leq\left\|\check{\zeta} \psi_{0}\right\|_{2}^{2} e^{-2 \gamma \kappa^{2} t}+\frac{C(\delta)}{\kappa^{4}}+\int_{0}^{t} e^{-2 \gamma \kappa^{2}(t-\tau)}\left|\left\langle\kappa B_{1, \kappa} \check{\zeta} \psi_{\kappa}, \check{\zeta} \psi_{\kappa}\right\rangle(\tau)\right| d \tau$.

Multiplying (4.1a) by $\check{\zeta}^{2} \bar{\psi}$ and integrating by parts yields $\frac{1}{2} \frac{d}{d t}\left(\left\|\check{\zeta} \psi_{\kappa}(t, \cdot)\right\|_{2}^{2}\right)+\left\|\nabla_{\kappa A_{\kappa}(t, \cdot)}\left(\check{\zeta} \psi_{\kappa}(t, \cdot)\right)\right\|_{2}^{2} \leq \kappa^{2}\left\|\check{\zeta} \psi_{\kappa}(t, \cdot)\right\|_{2}^{2}+\left\|\psi_{\kappa}(t, \cdot) \nabla \check{\zeta}\right\|_{2}^{2}$.

By Theorem 2.9 in [5] we have

$$
\begin{aligned}
\left\|\nabla_{\kappa A_{\kappa}}\left(\check{\zeta} \psi_{\kappa}\right)\right\|_{2}^{2} & \geq\left\langle\kappa B_{\kappa}(t, \cdot) \check{\zeta} \psi_{\kappa}(t, \cdot), \check{\zeta} \psi_{\kappa}(t, \cdot)\right\rangle \\
& =\kappa^{2}\left\langle B_{n} \check{\zeta} \psi_{\kappa}, \check{\zeta} \psi_{\kappa}\right\rangle+\left\langle\kappa B_{1, \kappa} \check{\zeta} \psi_{\kappa}, \check{\zeta} \psi_{\kappa}\right\rangle \\
& \geq \kappa^{2}\left(1+\frac{\delta}{2}\right)\left\|\check{\zeta} \psi_{\kappa}\right\|_{2}^{2}+\left\langle\kappa B_{1, \kappa} \check{\zeta} \psi_{\kappa}, \check{\zeta} \psi_{\kappa}\right\rangle .
\end{aligned}
$$

We can thus write

$$
\begin{aligned}
& \frac{1}{2} \frac{d}{d t}\left(\left\|\check{\zeta} \psi_{\kappa}(t, \cdot)\right\|_{2}^{2}\right)+\kappa^{2}\left(\frac{\delta}{2}-\frac{\delta}{4}\right)\left\|\check{\zeta} \psi_{\kappa}(t, \cdot)\right\|_{2}^{2} \\
\leq & \left\|\psi_{\kappa}(t, \cdot) \nabla \eta\right\|_{2}^{2}+\left\|\tilde{\chi} \psi_{\kappa}(t, \cdot) \nabla \eta_{\delta}\right\|_{2}^{2}-\left\langle\kappa B_{1, \kappa}(t, \cdot) \check{\zeta} \psi_{\kappa}(t, \cdot), \check{\zeta} \psi_{\kappa}(t, \cdot)\right\rangle .
\end{aligned}
$$

By (4.16), for every $0<\delta \leq \delta_{0}$, we have

$$
\left\|\psi_{\kappa}(t, \cdot) \nabla \eta\right\|_{2}^{2}+\left\|\tilde{\chi} \psi_{\kappa}(t, \cdot) \nabla \eta_{\delta}\right\|_{2}^{2} \leq \frac{C}{\kappa^{2}}
$$

which when substituted into (4.55) yields

$\frac{1}{2} \frac{d}{d t}\left(\left\|\check{\zeta} \psi_{\kappa}(t, \cdot)\right\|_{2}^{2}\right)+\kappa^{2} \gamma\left\|\check{\zeta} \psi_{\kappa}(t, \cdot)\right\|_{2}^{2} \leq \frac{C}{\kappa^{2}}-\left\langle\kappa B_{1, \kappa}(t, \cdot) \check{\zeta} \psi_{\kappa}(t, \cdot), \check{\zeta} \psi_{\kappa}(t, \cdot)\right\rangle$, 
where $\gamma=\delta / 4$. We now get (4.54) from (4.56).

Step 2: Prove that, for all $n \geq 2$,

$$
\begin{aligned}
& \left\|\check{\zeta} \psi_{\kappa}(\tau, \cdot)\right\|_{L^{\infty}\left(t^{*}+n-1, t^{*}+n, L^{2}(\Omega)\right)}^{2} \\
\leq & C \kappa^{-(p-2) / p}\left\|\check{\zeta} \psi_{\kappa}(\tau, \cdot)\right\|_{L^{\infty}\left(t^{*}+n-2, t^{*}+n, L^{2}(\Omega)\right)}^{2}+\frac{C}{\kappa^{4}},
\end{aligned}
$$

where $C$ is independent of $\kappa$.

To prove (4.57) we need to estimate the last term on the right-handside of (4.54). To this end we first write

$$
\begin{aligned}
& \int_{0}^{t} e^{-2 \gamma \kappa^{2}(t-\tau)}\left|\left\langle\kappa B_{1, \kappa} \check{\zeta} \psi_{\kappa}, \check{\zeta} \psi_{\kappa}\right\rangle(\tau)\right| d \tau \\
& \leq \kappa\left(\int_{t-1}^{t} e^{-2 \gamma \kappa^{2}(t-\tau)}\left\|B_{1, \kappa}(\tau, \cdot)\right\|_{L^{\infty}\left(\omega_{\delta / 2, j}\right)} d \tau\right) \cdot\left\|\check{\zeta} \psi_{\kappa}\right\|_{L^{\infty}\left(t-1, t ; L^{2}(\Omega)\right)} \\
& \quad+\kappa \int_{0}^{t-1} e^{-2 \gamma \kappa^{2}(t-\tau)}\left\|B_{1, \kappa}(\tau, \cdot)\right\|_{1} d \tau\left\|\check{\zeta} \psi_{\kappa}\right\|_{L^{\infty}\left(0, t-1 ; L^{2}(\Omega)\right)} .
\end{aligned}
$$

For the last term on the right-hand-side we have in view of Remark 4.2, (3.41), (3.42), and (4.3) that

$$
\kappa \int_{0}^{t-1} e^{-2 \gamma \kappa^{2}(t-\tau)}\left\|B_{1, \kappa}(\tau, \cdot)\right\|_{1} d \tau\left\|\check{\zeta} \psi_{\kappa}\right\|_{L^{\infty}\left(0, t-1 ; L^{2}(\Omega)\right)} \leq C e^{-2 \gamma \kappa^{2}} e^{C \kappa} .
$$

Hence for sufficiently large $\kappa$ we have

$$
\begin{gathered}
\int_{0}^{t} e^{-2 \gamma \kappa^{2}(t-\tau)}\left|\left\langle\kappa B_{1, \kappa} \check{\zeta} \psi_{\kappa}, \check{\zeta} \psi_{\kappa}\right\rangle(\tau)\right| d \tau \leq \\
\kappa\left(\int_{t-1}^{t} e^{-2 \gamma \kappa^{2}(t-\tau)}\left\|B_{1, \kappa}(\tau, \cdot)\right\|_{L^{\infty}\left(\omega_{\delta / 2, j}\right)} d \tau\right) \cdot\left\|\check{\zeta} \psi_{\kappa}\right\|_{L^{\infty}\left(t-1, t ; L^{2}(\Omega)\right)}+C e^{-\gamma \kappa^{2}} .
\end{gathered}
$$

Since by Sobolev embeddings

$$
\left\|B_{1, \kappa}\right\|_{L^{p}\left(t-1, t, L^{\infty}\left(\omega_{\delta / 2, j}\right)\right)} \leq C\left\|B_{1, \kappa}\right\|_{L^{p}\left(t-1, t, W^{1, p}\left(\omega_{\delta / 2, j}\right)\right)},
$$

we can use (4.38) to obtain, for sufficiently large $t$ and $\kappa$, and for any 
$2<p \leq 12 / 5$

$$
\begin{aligned}
& \int_{t-1}^{t} e^{-2 \gamma \kappa^{2}(t-\tau)}\left\|B_{1, \kappa}(\tau, \cdot)\right\|_{L^{\infty}\left(\omega_{\delta / 2, j}\right)} d \tau \\
\leq & \left\|B_{1, \kappa}(\tau, \cdot)\right\|_{L^{p}\left(t-1, t ; L^{\infty}\left(\omega_{\delta / 2, j}\right)\right)}\left[\int_{t-1}^{t} e^{-\frac{2 p}{p-1} \gamma \kappa^{2}(t-\tau)} d \tau\right]^{\frac{p-1}{p}} \\
\leq & C \kappa^{-2(p-1) / p} .
\end{aligned}
$$

Substituting the above into (4.58) yields

$$
\begin{aligned}
& \int_{0}^{t} e^{-2 \gamma \kappa^{2}(t-\tau)}\left|\left\langle\kappa B_{1, \kappa} \check{\zeta} \psi_{\kappa}, \check{\zeta} \psi_{\kappa}\right\rangle\right| d \tau \\
\leq & C \kappa^{-(p-2) / p}\left\|\check{\zeta} \psi_{\kappa}\right\|_{L^{\infty}\left(t-1, t ; L^{2}(\Omega)\right)}+C e^{-\frac{1}{4} \delta \kappa^{2}},
\end{aligned}
$$

which, when substituted into (4.54), yields (4.57).

Step 3: Prove (4.53).

Let now

$$
b_{n}=\left\|\check{\zeta} \psi_{\kappa}(\tau, \cdot)\right\|_{L^{\infty}\left(t^{*}+n-1, t^{*}+n, L^{2}(\Omega)\right)}^{2} .
$$

From (4.57) we get that if $p=12 / 5$ then for sufficiently large $\kappa$ it holds that

$$
b_{n} \leq C\left(\kappa^{-1 / 6}\left(b_{n-1}+b_{n}\right)+1\right),
$$

where $C$ is independent of $\kappa$. For another constant $\widehat{C}$, we get for sufficiently large $\kappa$,

$$
b_{n} \leq \widehat{C}\left(\kappa^{-1 / 6} b_{n-1}+1\right) .
$$

This immediately implies, for $\kappa$ large enough so that $\widehat{C} \kappa^{-\frac{1}{6}} \leq \frac{1}{2}$, the upperbound

$$
\limsup b_{n} \leq C_{0},
$$

where $C_{0}$ is independent of $\kappa$. Consequently,

$$
\limsup _{t \rightarrow \infty} \int_{\omega_{\delta, j}} \exp \left(\delta^{\frac{1}{2}} \kappa d\left(x, \partial \omega_{\delta, j}\right)\right)\left|\psi_{\kappa}(t, x)\right|^{2} d x \leq C_{0},
$$

which readily yields (4.53). 


\section{$5 \quad$ Large domains}

The main goal of this section is to prove Proposition 1.4. To this end it is more convenient to consider (1.26) in a fixed domain. Assuming that $0 \in \Omega$ we set $\epsilon=1 / R$ (hence we have $\epsilon \ll 1$ ) and apply the transformation,

$$
\psi_{\epsilon}(\epsilon x)=\psi(x), \quad A(x)=\epsilon^{-1} A_{\epsilon}(\epsilon x), \quad \phi(x)=\phi_{\epsilon}(\epsilon x),
$$

If we write $y=\epsilon x$, we have:

$$
\operatorname{curl}_{x}^{2} A=\epsilon \operatorname{curl}_{y}^{2} A_{\epsilon} \quad ; \quad \nabla_{x} \phi=\epsilon \nabla_{y} \phi_{\epsilon} \quad ; \quad \nabla_{A} \psi=\epsilon \nabla_{\epsilon^{-2} A_{\epsilon}} \psi_{\epsilon},
$$

leading to the following system for $\left(\psi_{\epsilon}, A_{\epsilon}, \phi_{\epsilon}\right)$

$$
\begin{array}{lr}
\Delta_{\epsilon^{-2} A_{\epsilon}} \psi_{\epsilon}+\frac{\psi_{\epsilon}}{\epsilon^{2}}\left(1-\left|\psi_{\epsilon}\right|^{2}\right)-\frac{i}{\epsilon^{2}} \phi_{\epsilon} \psi_{\epsilon}=0 & \text { in } \Omega, \\
\operatorname{curl}^{2} A_{\epsilon}+\nabla \phi_{\epsilon}=\operatorname{Im}\left(\bar{\psi}_{\epsilon} \nabla_{\epsilon^{-2} A_{\epsilon}} \psi_{\epsilon}\right) & \text { in } \Omega, \\
\psi_{\epsilon}=0 & \text { on } \partial \Omega_{c}, \\
\nabla_{\epsilon^{-2} A_{\epsilon}} \psi_{\epsilon} \cdot \nu=0 & \text { on } \partial \Omega_{i}, \\
\frac{\partial \phi_{\epsilon}}{\partial \nu}=f(\epsilon) J & \text { on } \partial \Omega, \\
\frac{\partial \phi_{\epsilon}}{\partial \nu}=0 & \text { on } \partial \Omega_{i}, \\
f_{\partial \Omega} \operatorname{curl} A_{\epsilon}(x) d s=f(\epsilon) h_{e x} . &
\end{array}
$$

In the above

$$
f(\epsilon)=F(1 / \epsilon)=\epsilon^{-\alpha} .
$$

It follows from (1.10) that

$$
h_{j}=b_{j} \epsilon^{-\alpha}, \quad j=1,2,
$$

where $b_{j}$ is independent of $\epsilon$ for $j=1,2$.

We assume that $A_{\epsilon}$ is in the Coulomb gauge space (1.9), and suppose that a weak solution $\left(\psi_{\epsilon}, A_{\epsilon}, \phi_{\epsilon}\right) \in H^{1}(\Omega, \mathbb{C}) \times H^{1}\left(\Omega, \mathbb{R}^{2}\right) \times L^{2}(\Omega)$ exists. Proposition 1.4 can now be reformulated in the following way: 
Proposition 5.1. Let $\left(\psi_{\epsilon}, A_{\epsilon}, \phi_{\epsilon}\right)$ denote a solution of (5.2), and let $h$ be given by (1.13). Suppose that for some $0<\gamma<1$ and $\epsilon_{0}>0$ we have

$$
\epsilon^{-\gamma}<h, \quad \forall 0<\epsilon<\epsilon_{0} .
$$

Then, there exists a compact set $K \subset \Omega, C>0$, and $\alpha>0$, such that for any $0<\epsilon<\epsilon_{0}$ we have

$$
\int_{K}\left|\psi_{\epsilon}(x)\right|^{2} d x \leq C e^{-\alpha / \epsilon} .
$$

We split the proof of Proposition 5.1 into several steps, to each of them we dedicate a separate lemma. We begin by observing, as in Section 3, that

$$
\left\|\psi_{\epsilon}\right\|_{\infty} \leq 1
$$

Let

$$
A_{1, \epsilon}=A_{\epsilon}-\epsilon^{-\alpha} A_{n}, \quad \phi_{1, \epsilon}=\phi_{\epsilon}-\epsilon^{-\alpha} \phi_{n},
$$

Set further

$$
B_{1, \epsilon}=\operatorname{curl} A_{1, \epsilon} ; \quad B_{\epsilon}=\operatorname{curl} A_{\epsilon} .
$$

By $(5.2 \mathrm{~b})$ and (1.16a), we then have

$$
\begin{cases}\nabla_{\perp} B_{1, \epsilon}+\nabla \phi_{1, \epsilon}=\operatorname{Im}\left(\bar{\psi}_{\epsilon} \nabla_{\epsilon^{-2} A_{\epsilon}} \psi_{\epsilon}\right) & \text { in } \Omega \\ \frac{\partial \phi_{1, \epsilon}}{\partial \nu}=0 & \text { on } \partial \Omega \\ f_{\partial \Omega} B_{1, \epsilon}(x) d s=0 . & \end{cases}
$$

Note that since $\partial B_{1, \epsilon} / \partial \tau=\partial \phi_{1, \epsilon} / \partial \nu=0$ on $\partial \Omega$ we must have by (5.7c) that

$$
\left.B_{1, \epsilon}\right|_{\partial \Omega} \equiv 0 .
$$

We begin with the following auxiliary estimate.

Lemma 5.2. Let $w_{\epsilon}$ denote the solution of

$$
\left\{\begin{array}{lc}
\Delta w_{\epsilon}-\frac{1}{\epsilon^{2}}\left|\psi_{\epsilon}\right|^{2} w_{\epsilon}=0 & \text { in } \Omega, \\
w_{\epsilon}=B_{\epsilon}-1 & \text { on } \partial \Omega .
\end{array}\right.
$$


Under the assumptions on $J$ and $\Omega$ in (1.4)-(1.7) we have

$$
\left\|B_{\epsilon}-1-w_{\epsilon}\right\|_{\infty} \leq \frac{1}{2} \text {. }
$$

Furthermore, we have

$$
\left\|\nabla \phi_{\epsilon}\right\|_{2}+\left\|\phi_{\epsilon}\right\|_{\infty} \leq C \epsilon^{-\alpha} .
$$

Proof. As can be easily verified from (5.2a) we have (see in [2] (formula (2.4) in the case when $\left.\phi_{\epsilon}=0\right)$,

$$
\frac{1}{2} \Delta\left|\psi_{\epsilon}\right|^{2}=-\frac{\left|\psi_{\epsilon}\right|^{2}}{\epsilon^{2}}\left(1-\left|\psi_{\epsilon}\right|^{2}\right)+\left|\nabla_{\epsilon^{-2} A_{\epsilon}} \psi_{\epsilon}\right|^{2} .
$$

Furthermore, taking the curl of (5.2b) yields

$$
\Delta B_{\epsilon}-\frac{1}{\epsilon^{2}}\left|\psi_{\epsilon}\right|^{2} B_{\epsilon}=-\operatorname{Im}\left(\nabla_{\epsilon^{-2} A_{\epsilon}} \bar{\psi}_{\epsilon} \times \nabla_{\epsilon^{-2} A_{\epsilon}} \psi_{\epsilon}\right) .
$$

Note that

$$
\begin{aligned}
& \operatorname{curl} \operatorname{Im}\left(\bar{\psi}_{\epsilon} \nabla_{\epsilon^{-2} A_{\epsilon}} \psi_{\epsilon}\right) \\
= & \operatorname{Im}\left(\nabla \bar{\psi}_{\epsilon} \times \nabla_{\epsilon^{-2} A_{\epsilon}} \psi_{\epsilon}-i \epsilon^{-2} \bar{\psi}_{\epsilon} A_{\epsilon} \times \nabla \psi_{\epsilon}\right)-\frac{1}{\epsilon^{2}}\left|\psi_{\epsilon}\right|^{2} B_{\epsilon} \\
= & \operatorname{Im}\left(\nabla_{\epsilon^{-2} A_{\epsilon}} \bar{\psi}_{\epsilon} \times \nabla_{\epsilon^{-2} A_{\epsilon}} \psi_{\epsilon}\right)-\frac{1}{\epsilon^{2}}\left|\psi_{\epsilon}\right|^{2} B_{\epsilon} .
\end{aligned}
$$

Let

$$
u_{\epsilon}=B_{\epsilon}-1+\frac{\left|\psi_{\epsilon}\right|^{2}}{2}-w_{\epsilon} .
$$

Combining the above and (5.12) yields that (cf. also [2])

$$
\begin{cases}\Delta u_{\epsilon}-\frac{1}{\epsilon^{2}}\left|\psi_{\epsilon}\right|^{2} u_{\epsilon}=\left|\nabla_{\epsilon^{-2} A_{\epsilon}} \psi_{\epsilon}\right|^{2}-\operatorname{Im}\left(\nabla_{\epsilon^{-2} A_{\epsilon}} \bar{\psi}_{\epsilon} \times \nabla_{\epsilon^{-2} A_{\epsilon}} \psi_{\epsilon}\right)+\frac{1}{2 \epsilon^{2}}\left|\psi_{\epsilon}\right|^{4} \geq 0 & \text { in } \Omega, \\ u_{\epsilon}=\frac{\left|\psi_{\epsilon}\right|^{2}}{2} & \text { on } \partial \Omega .\end{cases}
$$

By the weak maximum principle (cf. for instance [12, Theorem 8.1]) and (5.4) we obtain that for sufficiently small $\epsilon$

$$
u_{\epsilon}(x) \leq \frac{1}{2} .
$$


The lower bound in (5.10) follows easily by setting

$$
\tilde{u}_{\epsilon}=-B_{\epsilon}-1+\frac{\left|\psi_{\epsilon}\right|^{2}}{2}+w_{\epsilon}
$$

to obtain

$\begin{cases}\Delta \tilde{u}_{\epsilon}-\frac{1}{\epsilon^{2}}\left|\psi_{\epsilon}\right|^{2} \tilde{u}_{\epsilon}=\left|\nabla_{\epsilon^{-2} A_{\epsilon}} \psi_{\epsilon}\right|^{2}+\operatorname{Im}\left(\nabla_{\epsilon^{-2} A_{\epsilon}} \bar{\psi}_{\epsilon} \times \nabla_{\epsilon^{-2} A_{\epsilon}} \psi_{\epsilon}\right)+\frac{1}{2 \epsilon^{2}}\left|\psi_{\epsilon}\right|^{4} \geq 0 & \text { in } \Omega, \\ \tilde{u}_{\epsilon}=\frac{\left|\psi_{\epsilon}\right|^{2}}{2} & \text { on } \partial \Omega,\end{cases}$

upon which we use again the weak maximum principle.

To prove (5.11) we first obtain for $\phi_{\epsilon}$, in the same manner used to derive (3.7), the following problem:

$$
\left\{\begin{array}{rr}
-\Delta \phi_{\epsilon}+\frac{\rho_{\epsilon}^{2}}{\epsilon^{2}} \phi_{\epsilon}=0 & \text { in } \Omega, \\
\frac{\partial \phi_{\epsilon}}{\partial \nu}=\epsilon^{-\alpha} J & \text { on } \partial \Omega_{c}, \\
\frac{\partial \phi_{\epsilon}}{\partial \nu}=0 & \text { on } \partial \Omega_{i} .
\end{array}\right.
$$

Then, we follow the same steps as in the proof of (3.8) to obtain the bound $\left\|\phi_{\epsilon}\right\|_{\infty} \leq C \epsilon^{-\alpha}$. Multiplying (5.13) by $\phi_{\epsilon}$ and integrating by parts yields, using the preceding $L^{\infty}$ bound (5.10), we find that

$$
\left\|\nabla \phi_{\epsilon}\right\|_{2}^{2} \leq \int_{\partial \Omega} \phi_{\epsilon} \frac{\partial \phi_{\epsilon}}{\partial \nu} d s \leq C \epsilon^{-2 \alpha} .
$$

As a corollary we get:

\section{Corollary 5.3.}

$$
\left\|B_{\epsilon}\right\|_{\infty} \leq \max \left(\left|b_{1}\right|, b_{2}\right) \epsilon^{-\alpha}+\frac{1}{2} .
$$

Proof. By (5.10) and the maximum principle we have that

$$
\left\|B_{\epsilon}-1\right\|_{\infty} \leq\left\|w_{\epsilon}\right\|_{\infty}+\frac{1}{2} \leq \max \left(\left|b_{1}\right|, b_{2}\right) \epsilon^{-\alpha}-\frac{1}{2},
$$

which readily yields (5.14). 
We continue with the following auxiliary estimate:

Lemma 5.4. Let $\Omega$ and $J$ satisfy (1.4)-(1.7), and $w_{\epsilon}$ be a solution of (5.9). There exist positive constants $C$ and $\epsilon_{0}$, such that, for all $0<\epsilon<$ $\epsilon_{0}$,

$$
\left\|\nabla w_{\epsilon}\right\|_{\infty} \leq \frac{C}{\epsilon^{1+\alpha}}
$$

Proof. For convenience of notation we drop the subscript $\epsilon$ in the proof and bring only its main steps, as it rather standard. We first apply the inverse transformation of (5.1) to (5.9) to obtain

$$
\begin{cases}\Delta w-|\psi|^{2} w=0 & \text { in } \Omega_{R}, \\ w=B-1 & \text { on } \partial \Omega_{R},\end{cases}
$$

where $B=\operatorname{curl} A$.

We distinguish in the following between interior estimates and boundary estimates. Let $x_{0} \in \partial \Omega_{R}$ and $D_{r}=D_{r}\left(x_{0}\right)=B\left(x_{0}, r\right) \cap \Omega_{R}$.

By the standard elliptic estimates we then have, in view of (5.4),

$$
\|w\|_{H^{2}\left(D_{r}\right)} \leq C\left(\|w\|_{L^{2}\left(D_{2 r}\right)}+\left\|\epsilon^{-\alpha} B_{n}-1\right\|_{H^{2}\left(D_{2 r}\right)}\right) .
$$

To obtain the above we first observe that $B=\epsilon^{-\alpha} B_{n}$ on the boundary, and then use the fact that the trace of $B_{n}$ in $H^{\frac{3}{2}}(\partial \Omega)$ and is therefore bounded from above by a proper $H^{2}$ norm.

Similarly,

$$
\|w\|_{H^{3}\left(D_{r}\right)} \leq C\left(\|w\|_{H^{1}\left(D_{2 r}\right)}+\left\|\epsilon^{-\alpha} B_{n}-1\right\|_{H^{3}\left(D_{2 r}\right)}+\|w\|_{L^{\infty}\left(D_{2 r}\right)}\left\||\psi|^{2}\right\|_{H^{1}\left(D_{2 r}\right)}\right) .
$$

Using Kato's inequality and (1.26a) yields

$$
\|\nabla|\psi|\|_{L^{2}\left(D_{r}\right)} \leq\left\|\nabla_{A} \psi\right\|_{L^{2}\left(D_{r}\right)} \leq C\|\psi\|_{L^{2}\left(D_{2 r}\right)},
$$

then we obtain by (5.10) that

$$
\|w\|_{H^{3}\left(D_{r}\right)} \leq C \epsilon^{-\alpha}
$$

An interior estimate is even easier. Consider $x_{0} \in \Omega_{R}$ such that $D\left(x_{0}, 2 r\right) \subset \Omega_{R}$. There is no need in this case to include a boundary 
term in (5.16) and (5.17). We then obtain (5.18) in this case in a similar manner.

The proof of (5.15) now follows from Sobolev embeddings and (5.1).

We next define the following subdomain of $\Omega$ :

$$
\mathcal{D}_{\delta}(\epsilon)=\left\{x \in \Omega:\left|B_{\epsilon}(x)\right|<\delta \epsilon^{-\alpha}\right\} .
$$

Let further

$$
d_{\delta, j}(\epsilon)=d\left(\mathcal{D}_{\delta}(\epsilon), \partial \Omega_{i, j}\right), \quad j=1,2,
$$

where, as in the introduction, $\left\{\partial \Omega_{i, j}\right\}_{j=1}^{2}$ denotes the set of connected components of $\partial \Omega_{i}$. We now obtain a lower bound of

$$
d_{\delta}(\epsilon)=\max _{j \in\{1,2\}} d_{\delta, j}(\epsilon) .
$$

Lemma 5.5. Let

$$
\delta_{0}=\min \left(\left|b_{1}\right|,\left|b_{2}\right|\right) .
$$

Under the conditions of Lemma 5.4, there exists, for any $0<\delta<\delta_{0}$, a positive $C_{\delta}$ such that for sufficiently small $\epsilon$ we have

$$
d_{\delta}(\epsilon) \geq C_{\delta} \epsilon .
$$

Proof. Let $x \in \partial \Omega_{i}$ and $y \in \mathcal{D}_{\delta}(\epsilon)$. By (5.15) we have

$$
\left|w_{\epsilon}(x)-w_{\epsilon}(y)\right| \leq \frac{C}{\epsilon^{1+\alpha}}|x-y| .
$$

By (5.10) we have

$$
\left|w_{\epsilon}(x)-w_{\epsilon}(y)\right| \geq\left|B_{\epsilon}(x)-B_{\epsilon}(y)\right|-\frac{1}{2} \geq\left(\delta_{0}-\delta\right) \epsilon^{-\alpha}-\frac{1}{2} .
$$

Combining the above with (5.22) yields

$$
|x-y| \geq\left(\delta_{0}-\delta\right) \epsilon-\frac{1}{2} \epsilon^{1+\alpha},
$$

which readily yields (5.21). 
Next we show

Lemma 5.6. Under the conditions of Lemma 5.4, there exist $C>0$, $\epsilon_{0}>0$ and $\delta_{0}>0$ such that, for $0<\epsilon \leq \epsilon_{0}$ and $0<\delta \leq \delta_{0}$,

$$
\int_{\Omega \backslash \mathcal{D}_{\delta}(\epsilon)} \exp \left(\left[2 \delta \Theta_{0} \epsilon^{-\alpha}\right]^{1 / 2}(4 \epsilon)^{-1} d\left(x, \mathcal{D}_{\delta}(\epsilon)\right)\right)\left|\psi_{\epsilon}\right|^{2} d x \leq \frac{C}{\delta^{3 / 2}} .
$$

Proof. Let $\eta \in C^{\infty}(\Omega,[0,1])$ satisfy

$$
\eta(x)= \begin{cases}1 & x \in \Omega \backslash \mathcal{D}_{\delta}(\epsilon), \\ 0 & x \in \mathcal{D}_{\delta / 2}(\epsilon) .\end{cases}
$$

With the aid of (5.15) and (5.10), it can be easily verified, for some $C=C(p, J, \Omega)>0$ which is independent of both $\delta$ and $\epsilon$, that for all $0<\delta<\delta_{0}$ we can construct $\eta$ with the additional property

$$
|\nabla \eta| \leq \frac{C}{\delta \epsilon}
$$

Let further

$$
\zeta=\chi \eta
$$

where

$$
\chi= \begin{cases}\exp \left(\alpha_{\delta} \epsilon^{-1-\alpha / 2} d\left(x, \mathcal{D}_{\delta}(\epsilon)\right)\right) & \text { if } x \in \Omega \backslash \mathcal{D}_{\delta}(\epsilon), \\ 1 & \text { if } x \in \mathcal{D}_{\delta}(\epsilon)\end{cases}
$$

We leave the determination of $\alpha_{\delta}$ to a later stage.

Multiplying (3.1a) by $\zeta^{2} \bar{\psi}_{\epsilon}$ and integrating by parts yields

$$
\left\|\nabla_{\epsilon^{-2} A_{\epsilon}}\left(\zeta \psi_{\epsilon}\right)\right\|_{2}^{2}=\frac{1}{\epsilon^{2}}\left[\left\|\zeta \psi_{\epsilon}\right\|_{2}^{2}-\left\|\zeta^{1 / 2} \psi_{\epsilon}\right\|_{4}^{4}\right]+\left\|\psi_{\epsilon} \nabla \zeta\right\|_{2}^{2} .
$$

By (2.4) we have that for sufficiently small $\epsilon$

$$
\left\|\nabla_{\epsilon^{-2} A_{\epsilon}}\left(\zeta \psi_{\epsilon}\right)\right\|_{2}^{2} \geq \frac{\Theta_{0} \delta}{2 \epsilon^{2}} \epsilon^{-\alpha}\left\|\zeta \psi_{\epsilon}\right\|_{2}^{2},
$$

where $\Theta_{0}$ is defined in (2.2). Consequently,

$$
\frac{\Theta_{0} \delta \epsilon^{-\alpha}-2}{2 \epsilon^{2}}\left\|\zeta \psi_{\epsilon}\right\|_{2}^{2} \leq\left\|\psi_{\epsilon} \nabla \zeta\right\|_{2}^{2} .
$$


From (5.24) we learn that

$$
\left\|\zeta \psi_{\epsilon}\right\|_{2} \leq\left[\frac{2 \epsilon^{2}}{\Theta_{0} \delta \epsilon^{-\alpha}-2}\right]^{1 / 2}\left(\alpha_{\delta} \epsilon^{-(1+\alpha / 2)}\left\|\zeta \psi_{\epsilon}\right\|_{2}+\left\|\psi_{\epsilon} \nabla \eta\right\|_{2}\right)
$$

where we have used the fact that $\left|\nabla d\left(x, \mathcal{D}_{\delta}(\epsilon)\right)\right| \leq 1$ a.e.

Choosing

$$
\alpha_{\delta}=\frac{1}{2}\left[\frac{\Theta_{0} \delta}{2}\right]^{1 / 2}
$$

we obtain that for sufficiently small $\epsilon$

$$
\left\|\zeta \psi_{\epsilon}\right\|_{2} \leq 4\left[\frac{2}{\delta}\right]^{1 / 2} \epsilon^{1+\alpha / 2}\left\|\psi_{\epsilon} \nabla \eta\right\|_{2} \leq \frac{C \epsilon^{\alpha / 2}}{\delta^{3 / 2}}\left\|\psi_{\epsilon}\right\|_{L^{2}\left(\mathcal{D}_{\delta}(\epsilon) \backslash \mathcal{D}_{\delta / 2}(\epsilon)\right)} .
$$

The proof of (5.23) now follows from (5.4).

We now obtain an improved lower bound.

Proposition 5.7. Let $\delta_{0}$ be given by (5.20). Under the conditions of Lemma 5.4, for any $0<\delta<\delta_{0}$, there exist positive constants $C_{\delta}$ and $\epsilon_{\delta}$ such that for $0<\epsilon \leq \epsilon_{\delta}$ we have

$$
d_{\delta}(\epsilon) \geq C_{\delta} .
$$

Proof. We begin by noticing that by (5.2b), (5.11), and (5.23) we have

$$
\left\|\nabla B_{\epsilon}\right\|_{L^{2}\left(\Omega \backslash \mathcal{D}_{\delta}(\epsilon)\right)} \leq\left\|\nabla \phi_{\epsilon}\right\|_{L^{2}\left(\Omega \backslash \mathcal{D}_{\delta}(\epsilon)\right)}+C e^{-1 / \epsilon} \leq \widehat{C} \epsilon^{-\alpha} .
$$

Then, we write

$$
\left(\delta_{0}-\delta\right) \epsilon^{-\alpha} \leq\left\|\nabla B_{\epsilon}\right\|_{L^{1}\left(\Omega \backslash \mathcal{D}_{\delta}(\epsilon)\right)} \leq C d_{\delta}^{1 / 2}\left\|\nabla B_{\epsilon}\right\|_{L^{2}\left(\Omega \backslash \mathcal{D}_{\delta}(\epsilon)\right)} \leq C d_{\delta}^{1 / 2} \epsilon^{-\alpha},
$$

from which (5.25) readily follows.

Remark 5.8. Note that by (5.23) and the above arguments, (5.25) holds even if we use $d_{\delta}$ to measure the distance of $\mathcal{D}_{\delta}(\epsilon)$ from any point on $\partial \Omega$ where $B_{\epsilon}>\delta_{1} \epsilon^{-\alpha}$ with $\delta_{1}>\delta$.

Proposition 5.1 now follows from (5.23) and (5.25). 
Acknowledgements The authors gratefully acknowledge Phuc Nguyen for introducing to them Byun's result [8] and Monique Dauge for the idea behind the proof of Lemma 2.1. Y. Almog was partially supported by NSF grant DMS-1109030 and by US-Israel BSF grant no. 2010194. B. Helffer was partially supported by the ANR programme NOSEVOL and Simons foundation visiting fellow at the Newton Institute in Cambridge during the completion of this article. X.B. Pan was partially supported by the National Natural Science Foundation of China grant no. 11171111 and no. 11431005.

The authors declare that they have no conflict of interest.

\section{References}

[1] S. Agmon, Lectures on Elliptic Boundary Value Problems, Prepared for publication by B. Frank Jones, Jr. with the assistance of George W. Batten, Jr. Van Nostrand Mathematical Studies, No. 2, D. Van Nostrand Co., Inc., Princeton, N.J.-Toronto-London, 1965.

[2] Y. Almog, Non-linear surface superconductivity for type II superconductors in the large domain limit, Arch. Rat. Mech. Anal., 165 (2002), pp. 271-293.

[3] Y. Almog And B. Helffer, Global Stability of the Normal State of Superconductors in the Presence of a Strong Electric Current, Comm. Math. Phys., 330 (2014), pp. 1021-1094.

[4] K. ATtaR, The ground state energy of the two dimensional GinzburgLandau functional with variable magnetic field, Annales de l'Institut Henri Poincaré (C) Non Linear Analysis, Elsevier, (2014).

[5] Y. Avron, I. Herbst, And B. Simon, Schrödinger operators with magnetic fields I, Duke Math. J. 45 (1978), pp. 847-883.

[6] V. Bonnaillie, On the fundamental state energy for a Schrödinger operator with magnetic field in domains with corners, Asymptot. Anal., 41, 3-4 (2005), pp. 215-258. 
[7] V. Bonnaillie-Nö̈L And M. Dauge, Asymptotics for the lowlying eigenstates of the Schrödinger operator with magnetic field near corners, Ann. Henri Poincaré, 7 (2006), pp. 899-931.

[8] S.-S. Byun, Optimal $W^{1, p}$ regularity theory for parabolic equations in divergence form, J. Evol. Equ., 7 (2007), pp. 415-428.

[9] M. Dauge. Personal communication (2014).

[10] E. Feireisl And P. TAKÁČ, Long-time stabilization of solutions to the Ginzburg-Landau equations of superconductivity, Monatsh. Math., 133 (2001), pp. 197-221.

[11] S. Fournais and B. Helffer, Spectral Methods in Surface Superconductivity, Birkhäuser, 2009.

[12] D. Gilbarg and N. Trudinger, Elliptic Partial Differential Equations of Second Order, 2nd edition, Springer, Berlin, 1983.

[13] M. Giaquinta and L. Martinazzi, An introduction to the regularity theory for elliptic systems, harmonic maps and minimal graphs, vol. 11 of Appunti. Scuola Normale Superiore di Pisa (Nuova Serie) [Lecture Notes. Scuola Normale Superiore di Pisa (New Series)], Edizioni della Normale, Pisa, second ed., 2012.

[14] B. Helffer and A. Kachmar, The Ginzburg-Landau functional with vanishing magnetic field. to appear Arch. Rat. Mech. Anal., 2014.

[15] B. Helffer and A. Mohamed, Semiclassical analysis for the ground state energy of a Schrödinger operator with magnetic wells, J. Funct. Anal., 138 (1996), pp. 40-81.

[16] G. M. Lieberman, Second order parabolic differential equations, World Scientific Publishing Co., Inc., River Edge, NJ, 1996.

[17] F. H. Lin AND Q. Du, Ginzburg-Landau vortices: dynamics, pinning, and hysteresis, SIAM J. Math. Anal., 28, (1997), pp. 1265-1293.

[18] K. Lu AND X. PAN, Eigenvalue problems of Ginzburg-Landau operator in bounded domains, J. Math. Phys., 50 (1999), pp. 2647-2670. 
[19] L. Nirenberg, On elliptic partial differential equations, Ann. Scuola Norm. Sup. Pisa (3), 13 (1959), pp. 115-162.

[20] X. PAN, Upper critical for superconductors with edges and corners, Calc. Var. PDE, 14 (4) (2002), pp. 447-482.

[21] C. P. J. Poole, H. A. Farach, and R. J. Creswick, Handbook of Superconductivity, Academic Press, 1999.

[22] E. SAndier And S. Serfaty, The decrease of bulksuperconductivity near the second critical field in the GinzburgLandau model, SIAM J. Math. Anal., 34 (2003), pp. 939-956. 